\title{
Convergence Theorems of a General Composite Iterative Method for Nonexpansive Semigroups in Banach Spaces
}

\author{
Pitipong Sunthrayuth, ${ }^{1}$ Kriengsak Wattanawitoon, $^{2}$ \\ and Poom Kumam ${ }^{1}$ \\ ${ }^{1}$ Department of Mathematics, Faculty of Science, King Mongkut's University of Technology Thonburi \\ (KMUTT), Bangmod, Bangkok 10140, Thailand \\ ${ }^{2}$ Department of Mathematics, Faculty of Science and Agricultural Technology, \\ Rajamangala University of Technology Lanna Tak, Tak 63000, Thailand
}

Correspondence should be addressed to Poom Kumam, poom.kum@kmutt.ac.th

Received 9 December 2010; Accepted 10 January 2011

Academic Editor: G. Garcea

Copyright (C) 2011 Pitipong Sunthrayuth et al. This is an open access article distributed under the Creative Commons Attribution License, which permits unrestricted use, distribution, and reproduction in any medium, provided the original work is properly cited.

\begin{abstract}
We introduce a general composite iterative scheme for nonexpansive semigroups in Banach spaces. We establish some strong convergence theorems of the general iteration scheme under different control conditions. The results presented in this paper improve and extend the corresponding results of Marino and Xu (2006), and others, from Hilbert spaces to Banach spaces.
\end{abstract}

\section{Introduction}

Let $X$ be a real Banach space, and let $C$ be a nonempty closed convex subset of $X$. A mapping $T$ of $C$ into itself is said to be nonexpansive if $\|T x-T y\| \leq\|x-y\|$ for each $x, y \in C$. We denote $F(T)$ the set of fixed points of $T$. We know that $F(T)$ is nonempty if $C$ is bounded, for more detail see [1]. A one-parameter family $\tau=\{T(t): 0 \leq t<\infty\}$ from $C$ of $X$ into itself is said to be a nonexpansive semigroup on $C$ if it satisfies the following conditions:

(i) $T(0) x=x$ for all $x \in C$;

(ii) $T(s+t)=T(s) \circ T(t)$ for all $s, t \geq 0$;

(iii) for each $x \in C$ the mapping $t \mapsto T(t) x$ is continuous;

(iv) $\|T(t) x-T(t) y\| \leq\|x-y\|$ for all $x, y \in C$ and $t \geq 0$. 
We denote by $F(\tau)$ the set of all common fixed points of $\tau$, that is $F(\tau)=\bigcap_{t \geq 0} F(T(t))$. We know that $F(\tau)$ is nonempty if $C$ is bounded, see [2]. Recall that a self-mapping $f: C \rightarrow C$ is a contraction if there exists a constant $\alpha \in(0,1)$ such that $\|f(x)-f(y)\| \leq \alpha\|x-y\|$ for each $x, y \in C$. As in [3], we use the notation $\Pi_{C}$ to denote the collection of all contraction on $C$, that is, $\Pi_{C}=\{f: C \rightarrow C$ a contraction $\}$. Note that each $f \in \Pi_{C}$ has a unique fixed point in $C$. Iterative methods for nonexpansive mappings have recently been applied to solve minimization problems; see, for example, [4-10] and references therein.

A typical problem is to minimize a quadratic function over the set of the fixed points of a nonexpansive mapping on a real Hilbert space $H$ :

$$
\min _{x \in F} \frac{1}{2}\langle A x, x\rangle-\langle x, u\rangle
$$

where $F$ is the fixed point set of a nonexpansive mapping $T$ on $H$ and $u$ is a given point in $H$. Assume $A$ is strongly positive; that is there is a constant $\bar{\gamma}$ such that $\langle A x, x\rangle \geq \bar{\gamma}\|x\|^{2}$ for all $x \in H$.

In 2003, $\mathrm{Xu}$ [7] proved that the sequence $\left\{x_{n}\right\}$ generated by

$$
\begin{gathered}
x_{0}=x \in H \text { chosen arbitrarily; } \\
x_{n+1}=\alpha_{n} u+\left(1-\alpha_{n}\right) T x_{n}, \quad \forall n \geq 0,
\end{gathered}
$$

converges strongly to the unique solution of the minimization problem (1.1) provided the sequence $\left\{\alpha_{n}\right\}$ satisfies certain conditions. On the other hand, Moudafi [11] introduced the viscosity approximation method and proved that if $H$ is a real Hilbert space, the sequence $\left\{x_{n}\right\}$ generated by the following algorithm:

$$
\begin{aligned}
x_{0} & =x \in H \text { chosen arbitrarily; } \\
x_{n+1} & =\alpha_{n} f\left(x_{n}\right)+\left(1-\alpha_{n}\right) T x_{n}, \quad \forall n \geq 0,
\end{aligned}
$$

where $f: C \rightarrow C$ is a contraction mapping with constant $\alpha \in(0,1)$ and $\left\{\alpha_{n}\right\} \subset(0,1)$ satisfies certain conditions, converges strongly to a fixed point of $T$ in $C$ which is the unique solution of the following variational inequality:

$$
\left\langle(f-I) x^{*}, y-x^{*}\right\rangle \leq 0, \quad \forall y \in F(T)
$$

Recently, Marino and Xu [12] combined the iterative method (1.2) with the viscosity approximation method (1.3) considering the following general iterative process:

$$
\begin{gathered}
x_{0}=x \in H \text { chosen arbitrarily; } \\
x_{n+1}=\alpha_{n} \gamma f\left(x_{n}\right)+\left(I-\alpha_{n} A\right) T x_{n}, \quad \forall n \geq 0 .
\end{gathered}
$$


They proved the sequence $\left\{x_{n}\right\}$, generated by (1.5), converges strongly to the unique solution of the variational inequality

$$
\left\langle(\gamma f-A) x^{*}, y-x^{*}\right\rangle \leq 0, \quad \forall y \in F(T)
$$

which is the optimality condition for the minimization problem

$$
\min _{x \in C} \frac{1}{2}\langle A x, x\rangle-h(x)
$$

where $C$ is the fixed point set of a nonexpansive mapping $T$ and $h$ is a potential function for $\gamma f$ (i.e., $h^{\prime}(x)=\gamma f(x)$ for all $x \in H$ ). Xu [3] studied further the viscosity approximation method for nonexpansive semigroup in uniformly smooth Banach spaces. This result extends Theorem 2.2 of Moudafi [11] to a Banach space. Kim and $\mathrm{Xu}$ [13] studied the sequence generated by the algorithm

$$
\begin{gathered}
x_{1}=x \in C \text { chosen arbitrarily; } \\
y_{n}=\alpha_{n} x_{n}+\left(1-\alpha_{n}\right) T x_{n} ; \\
x_{n+1}=\beta_{n} u+\left(1-\beta_{n}\right) y_{n}, \quad \forall n \geq 1,
\end{gathered}
$$

and proved strong convergence of scheme (1.8) in the framework of a uniformly smooth Banach space. Later, Yao et al. [14] introduced a new iteration process by combining the modified Mann iteration [13] and the viscosity method introduced by Moudafi [11]. Let $C$ be a closed convex subset of a Banach space, $T: C \rightarrow C$ a nonexpansive mapping such that $F(T) \neq \emptyset$; and $f \in \Pi_{C}$. Define $\left\{x_{n}\right\}$ in the following way:

$$
\begin{gathered}
x_{1}=x \in C \text { chosen arbitrarily; } \\
y_{n}=\alpha_{n} x_{n}+\left(1-\alpha_{n}\right) T x_{n} ; \\
x_{n+1}=\beta_{n} f\left(x_{n}\right)+\left(1-\beta_{n}\right) y_{n}, \quad \forall n \geq 1,
\end{gathered}
$$

where $\left\{\alpha_{n}\right\}$ and $\left\{\beta_{n}\right\}$ are two sequences in $(0,1)$. They proved, under different control conditions on the sequences $\left\{\alpha_{n}\right\}$ and $\left\{\beta_{n}\right\}$, that $\left\{x_{n}\right\}$ converge strongly to a fixed point of $T$.

In 2008, Sahu and O'Regan [15] studied several strong convergence theorems for a family of nonexpansive or pseudocontractive nonself-mappings in a reflexive strictly convex Banach space with a uniformly Gâteaux differentiable norm. Recently, Li and Gu [16] studied the sequence generated by the algorithm in Banach spaces, as follows:

$$
\begin{gathered}
x_{1}=x \in C \text { chosen arbitrarily; } \\
y_{n}=\alpha_{n} x_{n}+\left(1-\alpha_{n}\right) T\left(t_{n}\right) x_{n} ; \\
x_{n+1}=\beta_{n} f\left(x_{n}\right)+\left(1-\beta_{n}\right) y_{n}, \quad \forall n \geq 1,
\end{gathered}
$$


and they proved the sequence $\left\{x_{n}\right\}$ defined by (1.10) that, converges strongly to the unique solution of the variational inequality:

$$
\left\langle(f-I) x^{*}, J\left(y-x^{*}\right)\right\rangle \leq 0, \quad \forall y \in F(\tau)
$$

Very recently, Kumam and Wattanawitoon [5] introduced the following new composite explicit iterative schemes defined by given $x_{0}=x \in C$ and

$$
\begin{gathered}
x_{n+1}=\alpha_{n} \gamma f\left(x_{n}\right)+\beta_{n} x_{n}+\left(\left(1-\beta_{n}\right) I-\alpha_{n} A\right) \frac{1}{t_{n}} \int_{0}^{t_{n}} T(s) y_{n} d s, \\
y_{n}=\delta_{n} \gamma f\left(x_{n}\right)+\gamma_{n} x_{n}+\left(\left(1-\gamma_{n}\right) I-\delta_{n} A\right) x_{n}, \quad \forall n \geq 0,
\end{gathered}
$$

for the approximation of common fixed point of a one parameter nonexpansive semigroup in a real Hilbert space under some appropriate control conditions. They proved strong convergence theorems of the composite iterative schemes which solve some variational inequalities under some appropriate conditions.

Question 1. Can the theorem of Marino and $\mathrm{Xu}$ [12] be extend from a Hilbert space to a general Banach space, such as uniformly smooth Banach space?

Question 2. Can we extend the iterative method of algorithm (1.10) to a general iterative process?

The purpose of this paper is to give affirmative answer to these questions mentioned above. In this paper, inspired and motivated by the iterative sequence (1.5) given by Marino and $\mathrm{Xu}$ [12] and (1.10) given by $\mathrm{Li}$ and $\mathrm{Gu}$ [16], we introduce a composite iterative algorithm $\left\{x_{n}\right\}$ in a Banach space as follows:

$$
\begin{gathered}
x_{1}=x \in C \text { chosen arbitrarily; } \\
y_{n}=\beta_{n} x_{n}+\left(1-\beta_{n}\right) T\left(t_{n}\right) x_{n} ; \\
x_{n+1}=\alpha_{n} \gamma f\left(x_{n}\right)+\delta_{n} x_{n}+\left(\left(1-\delta_{n}\right) I-\alpha_{n} A\right) y_{n}, \quad \forall n \geq 1,
\end{gathered}
$$

where $f: C \rightarrow C$ is a contraction mapping, $T\left(t_{n}\right)$ is a nonexpansive semigroup and $A$ is a strongly positive linear bounded operator, and prove, under certain different control conditions on the sequences $\left\{\alpha_{n}\right\},\left\{\beta_{n}\right\}$, and $\left\{\delta_{n}\right\}$, that $\left\{x_{n}\right\}$ defined by (1.13) converges strongly to a common fixed point, which solves some variational inequality in Banach spaces. The results presented in this paper extend the corresponding results announced by Marino and $\mathrm{Xu}[12]$ and some others from Hilbert spaces to Banach spaces. 


\section{Preliminaries}

Throughout this paper, let $X$ be a real Banach space, $C$ be a closed convex subset of $X$. Let $J: X \rightarrow 2^{X^{*}}$ be a normalized duality mapping by

$$
J(x)=\left\{f^{*} \in X^{*}:\left\langle x, f^{*}\right\rangle=\|x\|^{2}=\left\|f^{*}\right\|^{2}\right\}
$$

where $X^{*}$ denotes the dual space of $X$ and $\langle\cdot, \cdot\rangle$ denotes the generalized duality paring. In the following, the notation $\rightarrow$ and $\rightarrow$ denote the weak and strong convergence, respectively. Also, a mapping $I: C \rightarrow C$ denotes the identity mapping.

The norm of a Banach space $X$ is said to be Gâteaux differentiable if the limit

$$
\lim _{t \rightarrow 0} \frac{\|x+t y\|-\|x\|}{t}
$$

exists for each $x, y \in C$ on the unit sphere $S(X)$ of $X$. In this case $X$ is smooth. Recall that the Banach space $X$ is said to be smooth if duality mapping $J$ is single valued. In a smooth Banach space, we always assume that $A$ is strongly positive (see [17]), that is, a constant $\bar{\gamma}>0$ with the property

$$
\langle A x, J(x)\rangle \geq \bar{\gamma}\|x\|^{2}, \quad\|a I-b A\|=\sup _{\|x\| \leq 1}\|\langle(a I-b A) x, J(x)\rangle\|, \quad a \in[0,1], b \in[-1,1] .
$$

Moreover, if for each $y$ in $S(X)$ the limit (2.2) is uniformly attained for $x \in S(X)$, we say that the norm $X$ is uniformly Gâteaux differentiable. The norm of $X$ is said to be Frêchet differentiable, if for each $x \in S(X)$, the limit (2.2) is attained uniformly for $y \in S(X)$. The norm of $X$ is said to be uniformly Frêchet differentiable (or $X$ is said to be uniformly smooth), the limit (2.2) is attained uniformly for $(x, y) \in S(X) \times S(X)$. A Banach space $X$ is said to be strictly convex if $\|x\|=\|y\|=1, x \neq y$ implies $\|x+y\| / 2<1$; uniformly convex if $\delta_{X}(\epsilon)>0$ for all $\epsilon>0$, where $\delta_{X}(\epsilon)$ is modulus of convexity of $X$ defined by

$$
\delta_{X}(\epsilon)=\inf \left\{1-\frac{\|x+y\|}{2}:\|x\| \leq 1,\|y\| \leq 1,\|x+y\| \geq \varepsilon\right\}, \quad \forall \epsilon \in[0,2] .
$$

A uniformly convex Banach space $X$ is reflexive and strictly convex (see [18, Theorems 4.1.6 and 4.1.2]) and every uniformly smooth Banach space $X$ is a reflexive Banach with uniformly Gâteaux differentiable norm (see [18, Theorems 4.3.7 and 4.1.6]) (also see [19]).

Now, we present the concept of a uniformly asymptotically regular semigroup (see [20-22]). Let $C$ be a nonempty closed convex subset of a Banach space $X, \tau=\{T(t): t>0\}$ is a continuous operator semigroup on $C$. Then $\tau$ is said to uniformly asymptotically regular (in short, u.a.r.) on $C$ if for all $h \geq 0$ and any bounded subset $B$ of $C$,

$$
\limsup _{t \rightarrow \infty}\|T(h) T(t) x-T(t) x\|=0 .
$$


Lemma 2.1 (Chen and Song [23]). Let $C$ be a nonempty closed convex subset of a uniformly Banach space $X, B$ a bounded closed convex subset of $C$. If we denote $\tau=\{T(t): t>0\}$ a nonexpansive semigroup on $C$ such that $F:=\bigcap_{t>0} F(T(t)) \neq \emptyset$. For all $h \geq 0$, the set $\sigma_{t}(x)=(1 / t) \int_{0}^{t} T(s) x d s$, then

$$
\limsup _{t \rightarrow \infty}\left\|\sigma_{t}(x)-T(h) \sigma_{t}(x)\right\|=0 .
$$

It is easy to check that the set $\left\{\sigma_{t}: t>0\right\}$ defined by Lemma 2.1 is a u.a.r. nonexpansive semigroup on $C$ (see [24] for more detail).

Lemma 2.2 (Cai and $\mathrm{Hu}[17])$. Assume that $A$ is a strongly positive linear bounded operator on a smooth Banach space $X$ with coefficient $\bar{\gamma}>0$ and $0<\rho \leq\|A\|^{-1}$. Then $\|I-\rho A\| \leq 1-\rho \bar{\gamma}$.

Lemma 2.3 (Suzuki [25]). Let $\left\{x_{n}\right\}$ and $\left\{y_{n}\right\}$ be bounded sequences in a Banach space $X$ and let $\left\{\beta_{n}\right\}$ be a sequence in $[0,1]$ with $0<\lim _{\inf }{ }_{n \rightarrow \infty} \beta_{n} \leq \limsup _{n \rightarrow \infty} \beta_{n}<1$. Suppose $x_{n+1}=$ $\left(1-\beta_{n}\right) y_{n}+\beta_{n} x_{n}$ for all integers $n \geq 0$ and $\lim \sup _{n \rightarrow \infty}\left(\left\|y_{n+1}-y_{n}\right\|-\left\|x_{n+1}-x_{n}\right\|\right) \leq 0$. Then, $\lim _{n \rightarrow \infty}\left\|y_{n}-x_{n}\right\|=0$.

If a Banach space $X$ admits a sequentially continuous duality mapping $J$ from weak topology to weak star topology, then by Lemma 1 of [26], we have that duality mapping $J$ is a single value. In this case, the duality mapping $J$ is said to be a weakly sequentially continuous duality mapping, that is, for each $\left\{x_{n}\right\} \subset X$ with $x_{n} \rightarrow x$, we have $J\left(x_{n}\right)-^{*} J(x)$ (see [26-28] for more details).

A Banach space $X$ is said to be satisfying Opial's condition if for any sequence $x_{n} \rightarrow x$ for all $x \in X$ implies

$$
\limsup _{n \rightarrow \infty}\left\|x_{n}-x\right\|<\limsup _{n \rightarrow \infty}\left\|x_{n}-y\right\| \quad \forall y \in X, \text { with } x \neq y \text {. }
$$

By Theorem 1 in [26], it is well known that if $X$ admits a weakly sequentially continuous duality mapping, then $X$ satisfies Opial's condition and $X$ is smooth.

Lemma 2.4 (Demiclosed principle (Jung [27])). Let $C$ be a nonempty closed convex subset of a reflexive Banach space $X$ which satisfies Opial's condition, and suppose $T: C \rightarrow X$ is nonexpansive. Then the mapping $I-T$ is demiclosed at zero, that is, $x_{n} \rightarrow x$ and $x_{n}-T x_{n} \rightarrow 0$ implies $x=T x$.

Lemma 2.5 (Liu [29]). Let $X$ be a real Banach space and $J: X \rightarrow 2^{X^{*}}$ be the normalized duality mapping. Then, for any $x, y \in X$, we have

$$
\|x+y\|^{2} \leq\|x\|^{2}+2\langle y, j(x+y)\rangle
$$

for all $j(x+y) \in J(x+y)$ with $x \neq y$. 
Lemma 2.6 (Aoyama et al. [30]). Let $\left\{s_{n}\right\}$ be a sequence of nonnegative real numbers, $\left\{a_{n}\right\} a$ sequence of $[0,1]$ with $\sum_{n=1}^{\infty} a_{n}=\infty,\left\{c_{n}\right\}$ a sequence of nonnegative real number with $\sum_{n=1}^{\infty} c_{n}<\infty$, and $\left\{b_{n}\right\}$ a sequence of real numbers with $\lim _{\sup _{n \rightarrow \infty}} b_{n} \leq 0$. Suppose that

$$
s_{n+1}=\left(1-a_{n}\right) s_{n}+a_{n} b_{n}+c_{n}
$$

for all $n \in \mathbb{N}$. Then $\lim _{n \rightarrow \infty} s_{n}=0$.

\section{Main Results}

We need following the lemma to prove our theorem.

Lemma 3.1. Let $C$ be a nonempty bounded closed convex subset of a reflexive, smooth Banach space $X$ with admits a weakly sequentially continuous duality mapping $J$ from $X$ to $X^{*}$ with $C \pm C \subset C$ and let $\tau=\{T(t): 0 \leq t<\infty\}$ be a nonexpansive semigroup on $C$ such that $F(\tau) \neq \emptyset$. Let $f: C \rightarrow C$ be a contraction mapping with a coefficient $\alpha \in(0,1)$, A be a strongly positive linear bounded operator with a coefficient $\bar{\gamma}>0$ such that $0<\gamma<\bar{\gamma} / \alpha$ and $\left\{\lambda_{t}\right\}_{0<t<1}$ be a net of positive real sequence such that $\lim _{t \rightarrow 0} \lambda_{t}=\infty$. Suppose that $\left\{T\left(\lambda_{t}\right)\right\}$ is a u.a.r. nonexpansive semigroup. Then the sequence $\left\{x_{t}\right\}$ define by

$$
x_{t}=\operatorname{tr} f\left(x_{t}\right)+(I-t A) T\left(\lambda_{t}\right) x_{t}
$$

converges strongly to the common fixed point $x^{*}$, where $x^{*}$ solves the variational inequality

$$
\left\langle\gamma f\left(x^{*}\right)-A x^{*}, J\left(y-x^{*}\right)\right\rangle \leq 0, \quad \forall y \in F(\tau) .
$$

Proof. Observe that for $t \in\left(0,\|A\|^{-1}\right)$. By Lemma 2.2, we have $\|I-t A\| \leq 1-t \bar{\gamma}$.

Firstly, we show that $\left\{x_{t}\right\}$ defined by (3.1) is well define. Define the mapping $S^{f}: C \rightarrow$ $C$ provided by $S^{f}:=t \gamma f+(I-t A) T\left(\lambda_{t}\right)$, for each $t \in(0,1)$. Then, for each $x, y \in C$ that

$$
\begin{aligned}
\left\|S^{f} x-S^{f} y\right\| & =\left\|\operatorname{tr}(f(x)-f(y))+(I-t A)\left(T\left(\lambda_{t}\right) x-T\left(\lambda_{t}\right) y\right)\right\| \\
& \leq \operatorname{tr} \alpha\|x-y\|+(1-t \bar{\gamma})\|x-y\| \\
& =(1-t(\bar{\gamma}-\gamma \alpha))\|x-y\|<\|x-y\| .
\end{aligned}
$$

This show that $S^{f}$ is a contraction mapping. Thus, by Banach's contraction principle guarantees that $S^{f}$ has a unique fixed point $x_{t} \in C$, that is, $x_{t}$ defined by (3.1) is well define.

Next, we show the uniqueness of a solution of the variational inequality (3.2). Supposing $x^{*}, \tilde{x} \in F(\tau)$ satisfy the inequality (3.2), we have

$$
\begin{aligned}
& \left\langle(\gamma f-A) \tilde{x}, J\left(x^{*}-\tilde{x}\right)\right\rangle \leq 0, \\
& \left\langle(\gamma f-A) x^{*}, J\left(\tilde{x}-x^{*}\right)\right\rangle \leq 0 .
\end{aligned}
$$


Adding up both equations of (3.4), we get that

$$
\begin{aligned}
0 & \geq\left\langle(\gamma f-A) \tilde{x}-(\gamma f-A) x^{*}, J\left(x^{*}-\tilde{x}\right)\right\rangle \\
& =\left\langle A\left(x^{*}-\tilde{x}\right), J\left(x^{*}-\tilde{x}\right)\right\rangle-\gamma\left\langle f\left(x^{*}\right)-f(\tilde{x}), J\left(x^{*}-\tilde{x}\right)\right\rangle \\
& \geq \bar{\gamma}\left\|x^{*}-\tilde{x}\right\|^{2}-\gamma\left\|f\left(x^{*}\right)-f(\tilde{x})\right\|\left\|J\left(x^{*}-\tilde{x}\right)\right\| \\
& \geq \bar{\gamma}\left\|x^{*}-\tilde{x}\right\|^{2}-\gamma \alpha\left\|x^{*}-\tilde{x}\right\|^{2} \\
& =(\bar{\gamma}-\gamma \alpha)\left\|x^{*}-\tilde{x}\right\|^{2} .
\end{aligned}
$$

Since $0<\gamma<\bar{\gamma} / \alpha$ this implies that $\bar{\gamma}-\gamma \alpha>0$, which is a contradiction. Hence $\tilde{x}=x^{*}$ and the uniqueness is proved.

Next, we show that $\left\{x_{t}\right\}$ is bounded. Indeed, for any $p \in F(\tau)$, we have

$$
\begin{aligned}
\left\|x_{t}-p\right\| & =\left\|t \gamma f\left(x_{t}\right)+(I-t A) T\left(\lambda_{t}\right) x_{t}-p\right\| \\
& =\left\|t\left(\gamma f\left(x_{t}\right)-A p\right)+(I-t A)\left(T\left(\lambda_{t}\right) x_{t}-p\right)\right\| \\
& \leq t\left\|\gamma f\left(x_{t}\right)-A p\right\|+\|I-t A\|\left\|T\left(\lambda_{t}\right) x_{t}-p\right\| \\
& \leq t\left\|\gamma f\left(x_{t}\right)-A p\right\|+(1-t \bar{\gamma})\left\|x_{t}-p\right\| \\
& \leq t\left\|\gamma\left(f\left(x_{t}\right)-f(p)\right)+\gamma f(p)-A p\right\|+(1-t \bar{\gamma})\left\|x_{t}-p\right\| \\
& \leq t\left(\gamma \alpha\left\|x_{t}-p\right\|+\|\gamma f(p)-A p\|\right)+(1-t \bar{\gamma})\left\|x_{t}-p\right\| \\
& =(1-t(\bar{\gamma}-\gamma \alpha))\left\|x_{t}-p\right\|+t\|\gamma f(p)-A p\| .
\end{aligned}
$$

It follows that $\left\|x_{t}-p\right\| \leq\|\gamma f(p)-A p\| /(\bar{\gamma}-\gamma \alpha)$. Hence $\left\{x_{t}\right\}$ is bounded.

Next, we show that $\left\|x_{t}-T(h) x_{t}\right\| \rightarrow 0$ as $t \rightarrow 0$. We observe that

$$
\begin{aligned}
\left\|x_{t}-T(h) x_{t}\right\| & \leq\left\|x_{t}-T\left(\lambda_{t}\right) x_{t}\right\|+\left\|T\left(\lambda_{t}\right) x_{t}-T(h) T\left(\lambda_{t}\right) x_{t}\right\|+\left\|T(h) T\left(\lambda_{t}\right) x_{t}-T(h) x_{t}\right\| \\
& \leq 2\left\|x_{t}-T\left(\lambda_{t}\right) x_{t}\right\|+\left\|T\left(\lambda_{t}\right) x_{t}-T(h) T\left(\lambda_{t}\right) x_{t}\right\|
\end{aligned}
$$

for all $h \geq 0$. On the other hand, we note that

$$
x_{t}-T\left(\lambda_{t}\right) x_{t}=t\left(\gamma f\left(x_{t}\right)-A T\left(\lambda_{t}\right) x_{t}\right)
$$

for all $t>0$. By assuming that $\lim _{t \rightarrow 0} \lambda_{t}=\infty$ and $\left\{T\left(\lambda_{t}\right)\right\}$ be a u.a.r. nonexpansive semigroup, then for all $h \geq 0$, we get

$$
\lim _{\lambda_{t} \rightarrow \infty}\left\|T\left(\lambda_{t}\right) x_{t}-T(h) T\left(\lambda_{t}\right) x_{t}\right\| \leq \lim _{\lambda_{t} \rightarrow \infty} \sup _{z \in C}\left\|T\left(\lambda_{t}\right) z-T(h) T\left(\lambda_{t}\right) z\right\|=0 .
$$

From (3.7)-(3.9), letting $t \rightarrow 0$, we get

$$
\lim _{t \rightarrow 0}\left\|x_{t}-T(h) x_{t}\right\|=0
$$


for all $h \geq 0$. Assume $\left\{t_{n}\right\}_{n=1}^{\infty} \subset(0,1)$ is such that $t_{n} \rightarrow 0$ as $n \rightarrow \infty$. Put $x_{n}:=x_{t_{n}}$ and $\lambda_{n}:=\lambda_{t_{n}}$. We will show that $\left\{x_{n}\right\}$ contains a subsequence converges strongly to $x^{*}$, where $x^{*} \in F(\mathcal{S})$. Since $\left\{x_{n}\right\}$ is bounded sequence and Banach space $X$ is reflexive, there exists a subsequence $\left\{x_{n_{j}}\right\}$ of $\left\{x_{n}\right\}$ which converges weakly to $x^{*} \in C$ as $j \rightarrow \infty$. Again since Banach space $X$ has a weakly sequentially continuous duality mapping satisfying Opial's condition. It follows by Lemma 2.4 and noting (3.10), we have $x^{*} \in F(\tau)$. For each $n \geq 1$, we note that

$$
\begin{aligned}
x_{n}-x^{*} & =t_{n} \gamma f\left(x_{n}\right)+\left(I-t_{n} A\right) T\left(\lambda_{n}\right) x_{n}-x^{*} \\
& =t_{n}\left(\gamma f\left(x_{n}\right)-A x^{*}\right)+\left(I-t_{n} A\right)\left(T\left(\lambda_{n}\right) x_{n}-x^{*}\right) .
\end{aligned}
$$

Thus, we have

$$
\begin{aligned}
\left\|x_{n}-x^{*}\right\|^{2} & =t_{n}\left\langle\gamma f\left(x_{n}\right)-A x^{*}, J\left(x_{n}-x^{*}\right)\right\rangle+\left\langle\left(I-t_{n} A\right)\left(T\left(\lambda_{n}\right) x_{n}-x^{*}\right), J\left(x_{n}-x^{*}\right)\right\rangle \\
& \leq t_{n}\left\langle\gamma f\left(x_{n}\right)-A x^{*}, J\left(x_{n}-x^{*}\right)\right\rangle+\left\|I-t_{n} A\right\|\left\|T\left(\lambda_{n}\right) x_{n}-x^{*}\right\|\left\|J\left(x_{n}-x^{*}\right)\right\| \\
& \leq t_{n}\left\langle\gamma f\left(x_{n}\right)-A x^{*}, J\left(x_{n}-x^{*}\right)\right\rangle+\left(1-t_{n} \bar{\gamma}\right)\left\|x_{n}-x^{*}\right\|^{2} .
\end{aligned}
$$

It follows that

$$
\begin{aligned}
\left\|x_{n}-x^{*}\right\|^{2} & \leq \frac{1}{\bar{\gamma}}\left\langle\gamma f\left(x_{n}\right)-A x^{*}, J\left(x_{n}-x^{*}\right)\right\rangle \\
& =\frac{1}{\bar{\gamma}}\left[\left\langle\gamma f\left(x_{n}\right)-\gamma f\left(x^{*}\right), J\left(x_{n}-x^{*}\right)\right\rangle+\left\langle\gamma f\left(x^{*}\right)-A x^{*}, J\left(x_{n}-x^{*}\right)\right\rangle\right] \\
& \leq \frac{1}{\bar{\gamma}}\left[\gamma \alpha\left\|x_{n}-x^{*}\right\|^{2}+\left\langle\gamma f\left(x^{*}\right)-A x^{*}, J\left(x_{n}-x^{*}\right)\right\rangle\right] .
\end{aligned}
$$

Hence,

$$
\left\|x_{n}-x^{*}\right\|^{2} \leq \frac{1}{\bar{\gamma}-\gamma \alpha}\left\langle\gamma f\left(x^{*}\right)-A x^{*}, J\left(x_{n}-x^{*}\right)\right\rangle .
$$

In particular, we have

$$
\left\|x_{n_{j}}-x^{*}\right\|^{2} \leq \frac{1}{\bar{\gamma}-\gamma \alpha}\left\langle\gamma f\left(x^{*}\right)-A x^{*}, J\left(x_{n_{j}}-x^{*}\right)\right\rangle .
$$

Since $\left\{x_{n}\right\}$ is bounded and the duality mapping $J$ is single-valued and weakly sequentially continuous from $X$ into $X^{*}$, it follows (3.15), we have $x_{n_{j}} \rightarrow x^{*}$ as $j \rightarrow \infty$.

Next, we show that $x^{*}$ solves the variational inequality (3.2). Since

$$
x_{t}=\operatorname{tr} f\left(x_{t}\right)+(I-t A) T\left(\lambda_{t}\right) x_{t},
$$


we derive that

$$
(\gamma f-A) x_{t}=-\frac{1}{t}(I-t A)\left(T\left(\lambda_{t}\right) x_{t}-x_{t}\right)
$$

Notice that

$$
\begin{aligned}
\left\langle\left(T\left(\lambda_{t}\right) x_{t}-x_{t}\right)-\left(T\left(\lambda_{t}\right) y-y\right), J\left(y-x_{t}\right)\right\rangle & \geq\left\|x_{t}-y\right\|^{2}-\left\|T\left(\lambda_{t}\right) x_{t}-T\left(\lambda_{t}\right) y\right\|\left\|x_{t}-y\right\| \\
& \geq\left\|x_{t}-y\right\|^{2}-\left\|x_{t}-y\right\|^{2} \\
& =0 .
\end{aligned}
$$

It follows that, for any $y \in F(\tau)$,

$$
\begin{aligned}
\left\langle(\gamma f-A) x_{t}, J\left(y-x_{t}\right)\right\rangle= & -\frac{1}{t}\left\langle(I-t A)\left(T\left(\lambda_{t}\right) x_{t}-x_{t}\right), J\left(y-x_{t}\right)\right\rangle \\
= & -\frac{1}{t}\left\langle\left(T\left(\lambda_{t}\right) x_{t}-x_{t}\right)-\left(T\left(\lambda_{t}\right) y-y\right), J\left(y-x_{t}\right)\right\rangle \\
& +\left\langle A\left(T\left(\lambda_{t}\right) x_{t}-x_{t}\right), J\left(y-x_{t}\right)\right\rangle \\
\leq & \left\langle A\left(T\left(\lambda_{t}\right) x_{t}-x_{t}\right), J\left(y-x_{t}\right)\right\rangle .
\end{aligned}
$$

Now, replacing $t$ and $\lambda_{t}$ with $t_{n_{j}}$ and $\lambda_{n_{j}}$, respectively in (3.19), and letting $j \rightarrow \infty$, noting (3.8), we obtain $\left\langle(\gamma f-A) x^{*}, J\left(y-x^{*}\right)\right\rangle \leq 0$. That is, $x^{*}$ is a solution of variational inequality (3.2). By uniqueness, as $x^{*}=\tilde{x}$, we have shown that each cluster point of the sequence $\left\{x_{t}\right\}$ is equal to $x^{*}$. Then, we conclude that $x_{t} \rightarrow x^{*}$ as $t \rightarrow 0$. This completes the proof.

Now, we prove the following theorem which is the main result of this paper.

Theorem 3.2. Let $C$ be a nonempty bounded closed convex subset of a uniformly smooth Banach space $X$ which admit a weakly sequentially continuous duality mapping from $X$ into $X^{*}$ with $C \pm C \subset C$ and $\tau=\{T(t): 0 \leq t<\infty\}$ be a nonexpansive semigroup on $C$ such that $F(\tau) \neq \emptyset$. Let $f: C \rightarrow C$ be a contraction mapping with a coefficient $\alpha \in(0,1)$ and $A$ be a strongly positive bounded linear operator with coefficient $\bar{\gamma}>0$ such that $0<\gamma<\bar{\gamma} / \alpha$. Let $\left\{\alpha_{n}\right\}_{n=1}^{\infty},\left\{\beta_{n}\right\}_{n=1}^{\infty},\left\{\delta_{n}\right\}_{n=1}^{\infty}$ be the sequences in $(0,1)$ and $\left\{t_{n}\right\}_{n=1}^{\infty}$ be a positive real divergent sequence such that $t_{n} \leq t_{n+1}$ for all $n \in \mathbb{N}$. Assume the following control conditions are hold:

$$
\begin{aligned}
& \text { (C1) } \lim _{n \rightarrow \infty} \alpha_{n}=0 \text { and } \sum_{n=1}^{\infty} \alpha_{n}=\infty ; \\
& \text { (C2) } \sum_{n=1}^{\infty}\left|\alpha_{n+1}-\alpha_{n}\right|<\infty, \sum_{n=1}^{\infty}\left|\beta_{n+1}-\beta_{n}\right|<\infty \text { and } \sum_{n=1}^{\infty}\left|\delta_{n+1}-\delta_{n}\right|<\infty ; \\
& \text { (C3) } \sum_{n=1}^{\infty} \sup _{z \in C}\left\{\left\|T(h) T\left(t_{n}\right) z-T\left(t_{n}\right) z\right\|\right\}<\infty \text { for all } h \geq 0 .
\end{aligned}
$$

Then the sequence $\left\{x_{n}\right\}$ defined by (1.13) converges strongly to the common fixed point $x^{*}$, where $x^{*}$ is the unique solution in $F(\tau)$ of the variational inequality (3.2). 
Proof. First, we show that $\left\{x_{n}\right\}$ is bounded. By the control condition (C1), we may assume, without loss of generality, that $\alpha_{n} \leq\left(1-\delta_{n}\right)\|A\|^{-1}$ for all $n \geq 0$. Since $A$ is a linear bounded operator on $X$, by (2.3), we have $\|A\|=\sup \{|\langle A u, J(u)\rangle|: u \in X,\|u\|=1\}$. Observe that

$$
\begin{aligned}
\left\langle\left(\left(1-\delta_{n}\right) I-\alpha_{n} A\right) u, J(u)\right\rangle & =1-\delta_{n}-\alpha_{n}\langle A u, J(u)\rangle \\
& \geq 1-\delta_{n}-\alpha_{n}\|A\| \\
& \geq 0,
\end{aligned}
$$

that is to say $\left(1-\delta_{n}\right) I-\alpha_{n} A$ is positive. It follows that

$$
\begin{aligned}
\left\|\left(1-\delta_{n}\right) I-\alpha_{n} A\right\| & =\sup \left\{\left\langle\left(\left(1-\delta_{n}\right) I-\alpha_{n} A\right) u, J(u)\right\rangle: u \in X,\|u\|=1\right\} \\
& =\sup \left\{1-\delta_{n}-\alpha_{n}\langle A u, J(u)\rangle: u \in X,\|u\|=1\right\} \\
& \leq 1-\delta_{n}-\alpha_{n} \bar{\gamma}
\end{aligned}
$$

For any $p \in F(\tau)$, we compute

$$
\begin{aligned}
\left\|y_{n}-p\right\|= & \left\|\beta_{n} x_{n}+\left(1-\beta_{n}\right) T\left(t_{n}\right) x_{n}-p\right\| \\
= & \left\|\beta_{n}\left(x_{n}-p\right)+\left(1-\beta_{n}\right)\left(T\left(t_{n}\right) x_{n}-p\right)\right\| \\
\leq & \beta_{n}\left\|x_{n}-p\right\|+\left(1-\beta_{n}\right)\left\|T\left(t_{n}\right) x_{n}-p\right\| \\
\leq & \beta_{n}\left\|x_{n}-p\right\|+\left(1-\beta_{n}\right)\left\|x_{n}-p\right\| \\
= & \left\|x_{n}-p\right\| \\
\left\|x_{n+1}-p\right\|= & \left\|\alpha_{n} \gamma f\left(x_{n}\right)+\delta_{n} x_{n}+\left(\left(1-\delta_{n}\right) I-\alpha_{n} A\right) y_{n}-p\right\| \\
= & \left\|\alpha_{n}\left(\gamma f\left(x_{n}\right)-A p\right)+\delta_{n}\left(x_{n}-p\right)+\left(\left(1-\delta_{n}\right) I-\alpha_{n} A\right)\left(y_{n}-p\right)\right\| \\
\leq & \alpha_{n}\left[\gamma\left\|f\left(x_{n}\right)-f(p)\right\|+\|\gamma f(p)-A p\|\right]+\delta_{n}\left\|x_{n}-p\right\| \\
& +\left\|\left(1-\delta_{n}\right) I-\alpha_{n} A\right\|\left\|y_{n}-p\right\| \\
\leq & \alpha_{n}\left[\gamma \alpha\left\|x_{n}-p\right\|+\|\gamma f(p)-A p\|\right]+\delta_{n}\left\|x_{n}-p\right\|+\left(1-\delta_{n}-\alpha_{n} \bar{\gamma}\right)\left\|x_{n}-p\right\| \\
= & {\left[1-(\bar{\gamma}-\gamma \alpha) \alpha_{n}\right]\left\|x_{n}-p\right\|+(\bar{\gamma}-\gamma \alpha) \alpha_{n} \frac{\|\gamma f(p)-A p\|}{\bar{\gamma}-\gamma \alpha} . }
\end{aligned}
$$

By induction, we get

$$
\left\|x_{n+1}-p\right\| \leq \max \left\{\left\|x_{1}-p\right\|, \frac{\|\gamma f(p)-A p\|}{\bar{\gamma}-\gamma \alpha}\right\}
$$

for $n \geq 1$. Hence $\left\{x_{n}\right\}$ is bounded, so are $\left\{y_{n}\right\},\left\{f\left(x_{n}\right)\right\}$ and $\left\{T\left(t_{n}\right) x_{n}\right\}$. 
Next, we show that $\lim _{n \rightarrow \infty}\left\|x_{n+1}-x_{n}\right\|=0$. From definition of $\left\{y_{n}\right\}$, observing that

$$
\begin{gathered}
y_{n}=\beta_{n} x_{n}+\left(1-\beta_{n}\right) T\left(t_{n}\right) x_{n} \\
y_{n+1}=\beta_{n+1} x_{n+1}+\left(1-\beta_{n+1}\right) T\left(t_{n+1}\right) x_{n+1} .
\end{gathered}
$$

We note that

$$
\begin{aligned}
y_{n+1}-y_{n} & =\beta_{n+1} x_{n+1}+\left(1-\beta_{n+1}\right) T\left(t_{n+1}\right) x_{n+1}-\beta_{n} x_{n}-\left(1-\beta_{n}\right) T\left(t_{n}\right) x_{n} \\
& =\left(1-\beta_{n+1}\right)\left(T\left(t_{n+1}\right) x_{n+1}-T\left(t_{n}\right) x_{n}\right)+\beta_{n+1}\left(x_{n+1}-x_{n}\right)+\left(\beta_{n+1}-\beta_{n}\right)\left(x_{n}-T\left(t_{n}\right) x_{n}\right) .
\end{aligned}
$$

It follows that

$$
\begin{aligned}
\left\|y_{n+1}-y_{n}\right\| \leq & \left(1-\beta_{n+1}\right)\left\|T\left(t_{n+1}\right) x_{n+1}-T\left(t_{n}\right) x_{n}\right\|+\beta_{n+1}\left\|x_{n+1}-x_{n}\right\| \\
& +\left|\beta_{n+1}-\beta_{n}\right|\left\|x_{n}-T\left(t_{n}\right) x_{n}\right\| .
\end{aligned}
$$

Now, we consider the first term on the right side of (3.2), we have

$$
\begin{aligned}
\left\|T\left(t_{n+1}\right) x_{n+1}-T\left(t_{n}\right) x_{n}\right\| & \leq\left\|T\left(t_{n+1}\right) x_{n+1}-T\left(t_{n+1}\right) x_{n}\right\|+\left\|T\left(t_{n+1}\right) x_{n}-T\left(t_{n}\right) x_{n}\right\| \\
& \leq\left\|x_{n+1}-x_{n}\right\|+\left\|T\left(t_{n+1}\right) x_{n}-T\left(t_{n}\right) x_{n}\right\| .
\end{aligned}
$$

Substituting (3.27) into (3.26), we get

$$
\begin{aligned}
\left\|y_{n+1}-y_{n}\right\| \leq & \left(1-\beta_{n+1}\right)\left\|x_{n+1}-x_{n}\right\|+\left(1-\beta_{n+1}\right)\left\|T\left(t_{n+1}\right) x_{n}-T\left(t_{n}\right) x_{n}\right\| \\
& +\beta_{n+1}\left\|x_{n+1}-x_{n}\right\|+\left|\beta_{n+1}-\beta_{n}\right|\left\|x_{n}-T\left(t_{n}\right) x_{n}\right\| \\
\leq & \left\|x_{n+1}-x_{n}\right\|+\left\|T\left(t_{n+1}\right) x_{n}-T\left(t_{n}\right) x_{n}\right\|+\left|\beta_{n+1}-\beta_{n}\right|\left\|x_{n}-T\left(t_{n}\right) x_{n}\right\| \\
= & \left\|x_{n+1}-x_{n}\right\|+\left\|T\left(t_{n+1}-t_{n}\right) T\left(t_{n}\right) x_{n}-T\left(t_{n}\right) x_{n}\right\|+\left|\beta_{n+1}-\beta_{n}\right|\left\|x_{n}-T\left(t_{n}\right) x_{n}\right\| \\
\leq & \left\|x_{n+1}-x_{n}\right\|+\sup _{z \in C}\left\|T\left(t_{n+1}-t_{n}\right) T\left(t_{n}\right) z-T\left(t_{n}\right) z\right\|+\left|\beta_{n+1}-\beta_{n}\right|\left\|x_{n}-T\left(t_{n}\right) x_{n}\right\| .
\end{aligned}
$$

Similarly, from definition of $\left\{x_{n}\right\}$, observing that

$$
\begin{gathered}
x_{n+1}=\alpha_{n} \gamma f\left(x_{n}\right)+\delta_{n} x_{n}+\left(\left(1-\delta_{n}\right) I-\alpha_{n} A\right) y_{n}, \\
x_{n+2}=\alpha_{n+1} \gamma f\left(x_{n+1}\right)+\delta_{n+1} x_{n+1}+\left(\left(1-\delta_{n+1}\right) I-\alpha_{n+1} A\right) y_{n+1} .
\end{gathered}
$$


We note that

$$
\begin{aligned}
x_{n+2}-x_{n+1}= & \alpha_{n+1} \gamma f\left(x_{n+1}\right)+\delta_{n+1} x_{n+1}+\left(\left(1-\delta_{n+1}\right) I-\alpha_{n+1} A\right) y_{n+1} \\
& -\alpha_{n} \gamma f\left(x_{n}\right)-\delta_{n} x_{n}-\left(\left(1-\delta_{n}\right) I-\alpha_{n} A\right) y_{n} \\
= & \left(\left(1-\delta_{n+1}\right) I-\alpha_{n+1} A\right)\left(y_{n+1}-y_{n}\right)-\left(\alpha_{n+1}-\alpha_{n}\right) A y_{n}-\left(\delta_{n+1}-\delta_{n}\right) y_{n} \\
& +\left(\delta_{n+1} x_{n+1}-\delta_{n} x_{n}\right)+\gamma\left(\alpha_{n+1} f\left(x_{n+1}\right)-\alpha_{n} f\left(x_{n}\right)\right) \\
= & \left(\left(1-\delta_{n+1}\right) I-\alpha_{n+1} A\right)\left(y_{n+1}-y_{n}\right)+\left(\delta_{n+1} x_{n+1}-\delta_{n+1} x_{n}\right)+\gamma \alpha_{n+1}\left(f\left(x_{n+1}\right)-f\left(x_{n}\right)\right) \\
& +\left(\alpha_{n+1}-\alpha_{n}\right)\left(\gamma f\left(x_{n}\right)-A y_{n}\right)+\left(\delta_{n+1}-\delta_{n}\right)\left(x_{n}-y_{n}\right) .
\end{aligned}
$$

It follows that

$$
\begin{aligned}
\left\|x_{n+2}-x_{n+1}\right\| \leq & \left\|\left(1-\delta_{n+1}\right) I-\alpha_{n+1} A\right\|\left\|y_{n+1}-y_{n}\right\|+\delta_{n+1}\left\|x_{n+1}-x_{n}\right\|+\gamma \alpha_{n+1}\left\|f\left(x_{n+1}\right)-f\left(x_{n}\right)\right\| \\
& +\left|\alpha_{n+1}-\alpha_{n}\right|\left\|\gamma f\left(x_{n}\right)-A y_{n}\right\|+\left|\delta_{n+1}-\delta_{n}\right|\left\|x_{n}-y_{n}\right\| \\
\leq & \left(1-\delta_{n+1}-\alpha_{n+1} \bar{\gamma}\right)\left\|y_{n+1}-y_{n}\right\|+\delta_{n+1}\left\|x_{n+1}-x_{n}\right\|+\gamma \alpha \alpha_{n+1}\left\|x_{n+1}-x_{n}\right\| \\
& +\left|\alpha_{n+1}-\alpha_{n}\right|\left(\gamma\left\|f\left(x_{n}\right)\right\|+\left\|A y_{n}\right\|\right)+\left|\delta_{n+1}-\delta_{n}\right|\left\|x_{n}-y_{n}\right\| .
\end{aligned}
$$

Substituting (3.28) into (3.31), we get

$$
\begin{aligned}
\| x_{n+2} & -x_{n+1} \| \\
\leq & {\left[1-(\bar{\gamma}-\gamma \alpha) \alpha_{n+1}\right]\left\|x_{n+1}-x_{n}\right\|+\left(1-\delta_{n+1}-\alpha_{n+1} \bar{\gamma}\right) \sup _{z \in C}\left\|T\left(t_{n+1}-t_{n}\right) T\left(t_{n}\right) z-T\left(t_{n}\right) z\right\| } \\
& +\left(1-\delta_{n+1}-\alpha_{n+1} \bar{\gamma}\right)\left|\beta_{n+1}-\beta_{n}\right|\left\|x_{n}-T\left(t_{n}\right) x_{n}\right\|+\left|\alpha_{n+1}-\alpha_{n}\right|\left(\gamma\left\|f\left(x_{n}\right)\right\|+\left\|A y_{n}\right\|\right) \\
& +\left|\delta_{n+1}-\delta_{n}\right|\left(1-\beta_{n}\right)\left\|T\left(t_{n}\right) x_{n}-x_{n}\right\| \\
\leq & {\left[1-(\bar{\gamma}-\gamma \alpha) \alpha_{n+1}\right]\left\|x_{n+1}-x_{n}\right\|+\sup _{z \in C}\left\|T\left(t_{n+1}-t_{n}\right) T\left(t_{n}\right) z-T\left(t_{n}\right) z\right\| } \\
& +\left|\beta_{n+1}-\beta_{n}\right|\left\|x_{n}-T\left(t_{n}\right) x_{n}\right\|+\left|\alpha_{n+1}-\alpha_{n}\right|\left(\gamma\left\|f\left(x_{n}\right)\right\|+\left\|A y_{n}\right\|\right) \\
& +\left|\delta_{n+1}-\delta_{n}\right|\left\|T\left(t_{n}\right) x_{n}-x_{n}\right\| \\
\leq & {\left[1-(\bar{\gamma}-\gamma \alpha) \alpha_{n+1}\right]\left\|x_{n+1}-x_{n}\right\|+\sup _{z \in C}\left\|T\left(t_{n+1}-t_{n}\right) T\left(t_{n}\right) z-T\left(t_{n}\right) z\right\| } \\
& +M_{1}\left(\left|\alpha_{n+1}-\alpha_{n}\right|+\left|\beta_{n+1}-\beta_{n}\right|+\left|\delta_{n+1}-\delta_{n}\right|\right),
\end{aligned}
$$

where $M_{1}$ is an appropriate constant such that

$$
M_{1} \geq\left\|x_{n}-T\left(t_{n}\right) x_{n}\right\|+\gamma\left\|f\left(x_{n}\right)\right\|+\left\|A y_{n}\right\| \quad \forall n \in \mathbb{N} .
$$


Putting $c_{n}:=M_{1}\left(\left|\alpha_{n+1}-\alpha_{n}\right|+\left|\beta_{n+1}-\beta_{n}\right|+\left|\delta_{n+1}-\delta_{n}\right|\right)+\sup _{z \in C}\left\|T\left(t_{n+1}-t_{n}\right) T\left(t_{n}\right) z-T\left(t_{n}\right) z\right\|$, observing control conditions (C2) and (C3), we have

$$
\begin{aligned}
\sum_{n=1}^{\infty} c_{n}= & \sum_{n=1}^{\infty}\left(M_{1}\left(\left|\alpha_{n+1}-\alpha_{n}\right|+\left|\beta_{n+1}-\beta_{n}\right|+\left|\delta_{n+1}-\delta_{n}\right|\right)+\sup _{z \in C}\left\|T\left(t_{n+1}-t_{n}\right) T\left(t_{n}\right) z-T\left(t_{n}\right) z\right\|\right) \\
\leq & M_{1} \sum_{n=1}^{\infty}\left(\left|\alpha_{n+1}-\alpha_{n}\right|+\left|\beta_{n+1}-\beta_{n}\right|+\left|\delta_{n+1}-\delta_{n}\right|\right) \\
& +\sum_{n=1}^{\infty} \sup _{z \in C}\left\{\left\|T\left(t_{n+1}-t_{n}\right) T\left(t_{n}\right) z-T\left(t_{n}\right) z\right\|\right\}<\infty .
\end{aligned}
$$

Hence, by Lemma 2.6 to (3.32), we get that

$$
\lim _{n \rightarrow \infty}\left\|x_{n+1}-x_{n}\right\|=0
$$

Next, we show that

$$
\lim _{n \rightarrow \infty}\left\|T(h) x_{n}-x_{n}\right\|=0
$$

Observe that

$$
\begin{aligned}
\left\|y_{n}-x_{n}\right\| & \leq\left\|x_{n}-x_{n+1}\right\|+\left\|x_{n+1}-y_{n}\right\| \\
& =\left\|x_{n}-x_{n+1}\right\|+\left\|\alpha_{n} \gamma f\left(x_{n}\right)+\delta_{n}\left(x_{n}-y_{n}\right)-\alpha_{n} A y_{n}\right\| \\
& \leq\left\|x_{n}-x_{n+1}\right\|+\delta_{n}\left\|x_{n}-y_{n}\right\|+\alpha_{n}\left(\gamma\left\|f\left(x_{n}\right)\right\|+\left\|A y_{n}\right\|\right) .
\end{aligned}
$$

It follows that

$$
\left\|y_{n}-x_{n}\right\| \leq \frac{1}{1-\delta_{n}}\left(\left\|x_{n}-x_{n+1}\right\|+\alpha_{n}\left(\gamma\left\|f\left(x_{n}\right)\right\|+\left\|A y_{n}\right\|\right)\right)
$$

Observing control condition (C1) and noting (3.10), we have

$$
\lim _{n \rightarrow \infty}\left\|y_{n}-x_{n}\right\|=0
$$

Moreover, we note that

$$
\begin{aligned}
\left\|x_{n}-T\left(t_{n}\right) x_{n}\right\| & \leq\left\|x_{n}-y_{n}\right\|+\left\|y_{n}-T\left(t_{n}\right) x_{n}\right\| \\
& =\left\|x_{n}-y_{n}\right\|+\beta_{n}\left\|x_{n}-T\left(t_{n}\right) x_{n}\right\| .
\end{aligned}
$$


It follows that

$$
\left\|x_{n}-T\left(t_{n}\right) x_{n}\right\| \leq \frac{1}{1-\beta_{n}}\left\|x_{n}-y_{n}\right\|
$$

Noting (3.19), hence

$$
\lim _{n \rightarrow \infty}\left\|x_{n}-T\left(t_{n}\right) x_{n}\right\|=0
$$

On the other hand, we note that

$$
\begin{aligned}
\left\|T(h) x_{n}-x_{n}\right\| & \leq\left\|T(h) x_{n}-T(h) T\left(t_{n}\right) x_{n}\right\|+\left\|T(h) T\left(t_{n}\right) x_{n}-T\left(t_{n}\right) x_{n}\right\|+\left\|T\left(t_{n}\right) x_{n}-x_{n}\right\| \\
& \leq 2\left\|x_{n}-T\left(t_{n}\right) x_{n}\right\|+\left\|T(h) T\left(t_{n}\right) x_{n}-T\left(t_{n}\right) x_{n}\right\| \\
& \leq 2\left\|x_{n}-T\left(t_{n}\right) x_{n}\right\|+\sup _{z \in C}\left\|T(h) T\left(t_{n}\right) z-T\left(t_{n}\right) z\right\| .
\end{aligned}
$$

Therefor, by (3.26) and control condition (C3) imply that $\lim _{n \rightarrow \infty} \sup _{z \in C} \| T(h) T\left(t_{n}\right) z-$ $T\left(t_{n}\right) z \|=0$, we have

$$
\lim _{n \rightarrow \infty}\left\|T(h) x_{n}-x_{n}\right\|=0
$$

Next, we show that

$$
\limsup _{n \rightarrow \infty}\left\langle\gamma f\left(x^{*}\right)-A x^{*}, J\left(x_{n}-x^{*}\right)\right\rangle \leq 0
$$

For each $t \in(0,1)$, let $x_{t}$ be a unique point of $C$ such that $x_{t}=t \gamma f\left(x_{t}\right)+(I-t A) T\left(\lambda_{t}\right) x_{t}$. By Lemma 3.1, we have

$$
\begin{aligned}
\left\|x_{t}-x_{n}\right\|^{2} \leq & \left\|(I-t A)\left(T\left(\lambda_{t}\right) x_{t}-x_{n}\right)\right\|^{2}+2 t\left\langle\gamma f\left(x_{t}\right)-A x_{n}, J\left(x_{t}-x_{n}\right)\right\rangle \\
\leq & \|I-t A\|^{2}\left\|T\left(\lambda_{t}\right) x_{t}-x_{n}\right\|^{2}+2 t\left\langle\gamma f\left(x_{t}\right)-A x_{n}, J\left(x_{t}-x_{n}\right)\right\rangle \\
\leq & (1-t \bar{\gamma})^{2}\left(\left\|T\left(\lambda_{t}\right) x_{t}-T\left(\lambda_{t}\right) x_{n}\right\|+\left\|T\left(\lambda_{t}\right) x_{n}-x_{n}\right\|\right)^{2}+2 t\left\langle\gamma f\left(x_{t}\right)-A x_{n}, J\left(x_{t}-x_{n}\right)\right\rangle \\
\leq & \left(1-2 \bar{\gamma} t+\bar{\gamma}^{2} t^{2}\right)\left\|x_{t}-x_{n}\right\|^{2}+f_{n}(t)+2 t\left\langle\gamma f\left(x_{t}\right)-A x_{t}, J\left(x_{t}-x_{n}\right)\right\rangle \\
& +2 t\left\langle A x_{t}-A x_{n}, J\left(x_{t}-x_{n}\right)\right\rangle,
\end{aligned}
$$

where $f_{n}(t):=\left(2\left\|x_{t}-x_{n}\right\|+\left\|x_{n}-T\left(\lambda_{t}\right) x_{n}\right\|\right)\left\|x_{n}-T\left(\lambda_{t}\right) x_{n}\right\|$. 
On the other hand, observe that $A$ is a strongly positive linear bounded operator, it follows from (2.3), we have

$$
\left\langle A x_{t}-A x_{n}, J\left(x_{t}-x_{n}\right)\right\rangle=\left\langle A\left(x_{t}-x_{n}\right), J\left(x_{t}-x_{n}\right)\right\rangle \geq \bar{\gamma}\left\|x_{t}-x_{n}\right\|^{2} .
$$

Combining (3.47) with (3.46), we have

$$
\begin{aligned}
2 t\left\langle A x_{t}-\gamma f\left(x_{t}\right), J\left(x_{t}-x_{n}\right)\right\rangle \\
\leq\left(\bar{\gamma}^{2} t^{2}-2 \bar{\gamma} t\right)\left\|x_{t}-x_{n}\right\|^{2}+a_{n}(t)+2 t\left\langle A x_{t}-A x_{n}, J\left(x_{t}-x_{n}\right)\right\rangle \\
\leq\left(\bar{\gamma} t^{2}-2 t\right)\left\langle A x_{t}-A x_{n}, J\left(x_{t}-x_{n}\right)\right\rangle+2 t\left\langle A x_{t}-A x_{n}, J\left(x_{t}-x_{n}\right)\right\rangle+f_{n}(t) \\
\quad=\bar{\gamma} t^{2}\left\langle A x_{t}-A x_{n}, J\left(x_{t}-x_{n}\right)\right\rangle+f_{n}(t) .
\end{aligned}
$$

It follows that

$$
\left\langle A x_{t}-\gamma f\left(x_{t}\right), J\left(x_{t}-x_{n}\right)\right\rangle \leq \frac{\bar{\gamma} t}{2}\left\langle A x_{t}-A x_{n}, J\left(x_{t}-x_{n}\right)\right\rangle+\frac{1}{2 t} f_{n}(t) .
$$

Now, taking limit superior as $n \rightarrow \infty$ firstly, and then as $t \rightarrow 0$ in (3.49) (using (3.44)), we have

$$
\limsup _{n \rightarrow \infty}\left\langle A x_{t}-\gamma f\left(x_{t}\right), J\left(x_{t}-x_{n}\right)\right\rangle \leq \frac{t}{2} M_{2}
$$

where $M_{2}>0$ is a constant such that $M_{2} \geq \bar{\gamma}\left\langle A x_{t}-A x_{n}, J\left(x_{t}-x_{n}\right)\right\rangle$ for all $t \in(0,1)$ and $n \geq 1$. Now, taking limit superior as $t \rightarrow 0$ in (3.50). Hence, we get

$$
\limsup _{t \rightarrow 0} \limsup _{n \rightarrow \infty}\left\langle A x_{t}-\gamma f\left(x_{t}\right), J\left(x_{t}-x_{n}\right)\right\rangle \leq 0 .
$$

Moreover, we note that

$$
\begin{aligned}
\left\langle\gamma f\left(x^{*}\right)\right. & \left.-A x^{*}, J\left(x_{n}-x^{*}\right)\right\rangle \\
= & \left\langle\gamma f\left(x^{*}\right)-A x^{*}, J\left(x_{n}-x^{*}\right)\right\rangle-\left\langle\gamma f\left(x^{*}\right)-A x^{*}, J\left(x_{n}-x_{t}\right)\right\rangle \\
& +\left\langle\gamma f\left(x^{*}\right)-A x^{*}, J\left(x_{n}-x_{t}\right)\right\rangle-\left\langle\gamma f\left(x^{*}\right)-A x_{t}, J\left(x_{n}-x_{t}\right)\right\rangle \\
& +\left\langle\gamma f\left(x^{*}\right)-A x_{t}, J\left(x_{n}-x_{t}\right)\right\rangle-\left\langle\gamma f\left(x_{t}\right)-A x_{t}, J\left(x_{n}-x_{t}\right)\right\rangle \\
& +\left\langle\gamma f\left(x_{t}\right)-A x_{t}, J\left(x_{n}-x_{t}\right)\right\rangle \\
= & \left\langle\gamma f\left(x^{*}\right)-A x^{*}, J\left(x_{n}-x^{*}\right)-J\left(x_{n}-x_{t}\right)\right\rangle+\left\langle A x_{t}-A x^{*}, J\left(x_{n}-x_{t}\right)\right\rangle \\
& +\left\langle\gamma f\left(x^{*}\right)-\gamma f\left(x_{t}\right), J\left(x_{n}-x_{t}\right)\right\rangle+\left\langle\gamma f\left(x_{t}\right)-A x_{t}, J\left(x_{n}-x_{t}\right)\right\rangle .
\end{aligned}
$$


Taking limit superior as $n \rightarrow \infty$ in (3.52), we have

$$
\begin{aligned}
\limsup _{n \rightarrow \infty}\left\langle\gamma f\left(x^{*}\right)-A x^{*}, J\left(x_{n}-x^{*}\right)\right\rangle \leq & \limsup _{n \rightarrow \infty}\left\langle\gamma f\left(x^{*}\right)-A x^{*}, J\left(x_{n}-x^{*}\right)-J\left(x_{n}-x_{t}\right)\right\rangle \\
& +\|A\|\left\|x_{t}-x^{*}\right\| \limsup _{n \rightarrow \infty}\left\|x_{n}-x_{t}\right\| \\
& +\left\|\gamma f\left(x^{*}\right)-\gamma f\left(x_{t}\right)\right\| \limsup _{n \rightarrow \infty}\left\|x_{n}-x_{t}\right\| \\
& +\limsup _{n \rightarrow \infty}\left\langle\gamma f\left(x_{t}\right)-A x_{t}, J\left(x_{n}-x_{t}\right)\right\rangle \\
\leq & \limsup _{n \rightarrow \infty}\left\langle\gamma f\left(x^{*}\right)-A x^{*}, J\left(x_{n}-x^{*}\right)-J\left(x_{n}-x_{t}\right)\right\rangle \\
& +(\|A\|+\gamma \alpha)\left\|x_{t}-x^{*}\right\| \limsup _{n \rightarrow \infty}\left\|x_{n}-x_{t}\right\| \\
& +\limsup _{n \rightarrow \infty}\left\langle\gamma f\left(x_{t}\right)-A x_{t}, J\left(x_{n}-x_{t}\right)\right\rangle .
\end{aligned}
$$

By Lemma 3.1, $x_{t} \rightarrow x^{*} \in F(\tau)$ as $t \rightarrow 0$. Since $X$ is a uniformly smooth Banach space, imply that $J$ is norm-to-norm uniformly continuous on bounded subset of $C$ (see, e.g., [18, Lemma 1]), we obtain

$$
\limsup _{t \rightarrow 0} \limsup _{n \rightarrow \infty}\left\langle\gamma f\left(x^{*}\right)-A x^{*}, J\left(x_{n}-x^{*}\right)-J\left(x_{n}-x_{t}\right)\right\rangle=0 .
$$

Therefore, from (3.53), we have

$$
\begin{aligned}
\limsup _{n \rightarrow \infty}\left\langle\gamma f\left(x^{*}\right)-A x^{*}, J\left(x_{n}-x^{*}\right)\right\rangle & =\limsup _{t \rightarrow 0} \limsup _{n \rightarrow \infty}\left\langle\gamma f\left(x^{*}\right)-A x^{*}, J\left(x_{n}-x^{*}\right)\right\rangle \\
& \leq \limsup _{t \rightarrow 0} \limsup _{n \rightarrow \infty}\left\langle\gamma f\left(x_{t}\right)-A x_{t}, J\left(x_{n}-x_{t}\right)\right\rangle \\
& \leq 0 .
\end{aligned}
$$

Finally, we show that $\lim _{n \rightarrow \infty}\left\|x_{n}-x^{*}\right\|=0$. By Lemma 2.5, we have

$$
\begin{aligned}
\| x_{n+1} & -x^{*} \|^{2} \\
= & \left\|\alpha_{n} \gamma f\left(x_{n}\right)+\delta_{n} x_{n}+\left(\left(1-\delta_{n}\right) I-\alpha_{n} A\right) y_{n}-x^{*}\right\|^{2} \\
= & \left\|\left(\left(1-\delta_{n}\right) I-\alpha_{n} A\right)\left(y_{n}-x^{*}\right)+\delta_{n}\left(x_{n}-x^{*}\right)+\alpha_{n}\left(\gamma f\left(x_{n}\right)-A x^{*}\right)\right\|^{2} \\
\leq & \left\|\left(\left(1-\delta_{n}\right) I-\alpha_{n} A\right)\left(y_{n}-x^{*}\right)+\delta_{n}\left(x_{n}-x^{*}\right)\right\|^{2} \\
& +2 \alpha_{n}\left\langle\gamma f\left(x_{n}\right)-A x^{*}, J\left(x_{n+1}-x^{*}\right)\right\rangle
\end{aligned}
$$




$$
\begin{aligned}
\leq & {\left[\left\|\left(\left(1-\delta_{n}\right) I-\alpha_{n} A\right)\left(y_{n}-x^{*}\right)\right\|+\delta_{n}\left\|x_{n}-x^{*}\right\|\right]^{2} } \\
& +2 \alpha_{n}\left\langle\gamma f\left(x_{n}\right)-A x^{*}, J\left(x_{n+1}-x^{*}\right)\right\rangle \\
\leq & {\left[\left\|\left(1-\delta_{n}\right) I-\alpha_{n} A\right\|\left\|y_{n}-x^{*}\right\|+\delta_{n}\left\|x_{n}-x^{*}\right\|\right]^{2}+2 \alpha_{n}\left\langle\gamma f\left(x_{n}\right)-A x^{*}, J\left(x_{n+1}-x^{*}\right)\right\rangle } \\
\leq & {\left[\left\|1-\delta_{n}-\alpha_{n} \bar{\gamma}\right\|\left\|x_{n}-x^{*}\right\|+\delta_{n}\left\|x_{n}-x^{*}\right\|\right]^{2}+2 \alpha_{n}\left\langle\gamma f\left(x_{n}\right)-A x^{*}, J\left(x_{n+1}-x^{*}\right)\right\rangle } \\
\leq & \left(1-\alpha_{n} \bar{\gamma}\right)^{2}\left\|x_{n}-x^{*}\right\|^{2}+2 \alpha_{n}\left\langle\gamma f\left(x_{n}\right)-\gamma f\left(x^{*}\right), J\left(x_{n+1}-x^{*}\right)\right\rangle \\
& +2 \alpha_{n}\left\langle\gamma f\left(x^{*}\right)-A x^{*}, J\left(x_{n+1}-x^{*}\right)\right\rangle \\
\leq & \left(1-\alpha_{n} \bar{\gamma}\right)^{2}\left\|x_{n}-x^{*}\right\|^{2}+2 \alpha_{n} \gamma \alpha\left\|x_{n}-x^{*}\right\|\left\|x_{n+1}-x^{*}\right\|+2 \alpha_{n}\left\langle\gamma f\left(x^{*}\right)-A x^{*}, J\left(x_{n+1}-x^{*}\right)\right\rangle \\
\leq & \left(1-\alpha_{n} \bar{\gamma}\right)^{2}\left\|x_{n}-x^{*}\right\|^{2}+\alpha_{n} \gamma \alpha\left(\left\|x_{n}-x^{*}\right\|^{2}+\left\|x_{n+1}-x^{*}\right\|^{2}\right) \\
& +2 \alpha_{n}\left\langle\gamma f\left(x^{*}\right)-A x^{*}, J\left(x_{n+1}-x^{*}\right)\right\rangle .
\end{aligned}
$$

It follows that

$$
\begin{aligned}
\left\|x_{n+1}-x^{*}\right\|^{2} \leq & {\left[\frac{\left(1-\alpha_{n} \bar{\gamma}\right)^{2}+\alpha_{n} \gamma \alpha}{1-\alpha_{n} \gamma \alpha}\right]\left\|x_{n}-x^{*}\right\|^{2}+\frac{2 \alpha_{n}}{1-\alpha_{n} \gamma \alpha}\left\langle\gamma f\left(x^{*}\right)-A x^{*}, J\left(x_{n+1}-x^{*}\right)\right\rangle } \\
= & {\left[\frac{1-2 \alpha_{n} \bar{\gamma}+\alpha_{n} \gamma \alpha}{1-\alpha_{n} \gamma \alpha}\right]\left\|x_{n}-x^{*}\right\|^{2}+\frac{\alpha_{n}^{2} \bar{\gamma}^{2}}{1-\alpha_{n} \gamma \alpha}\left\|x_{n}-x^{*}\right\|^{2} } \\
& +\frac{2 \alpha_{n}}{1-\alpha_{n} \gamma \alpha}\left\langle\gamma f\left(x^{*}\right)-A x^{*}, J\left(x_{n+1}-x^{*}\right)\right\rangle \\
= & {\left[1-\frac{2 \alpha_{n}(\bar{\gamma}-\gamma \alpha)}{1-\alpha_{n} \gamma \alpha}\right]\left\|x_{n}-x^{*}\right\|^{2}+\frac{\alpha_{n}^{2} \bar{\gamma}^{2}}{1-\alpha_{n} \gamma \alpha}\left\|x_{n}-x^{*}\right\|^{2} } \\
& +\frac{2 \alpha_{n}}{1-\alpha_{n} \gamma \alpha}\left\langle\gamma f\left(x^{*}\right)-A x^{*}, J\left(x_{n+1}-x^{*}\right)\right\rangle .
\end{aligned}
$$

Put $a_{n}:=2 \alpha_{n}(\bar{\gamma}-\gamma \alpha) /\left(1-\alpha_{n} \gamma \alpha\right)$ and $b_{n}:=\left(\alpha_{n} \bar{\gamma}^{2} / 2(\bar{\gamma}-\gamma \alpha)\right)\left\|x_{n}-x^{*}\right\|^{2}+(1 /(\bar{\gamma}-\gamma \alpha))\left\langle\gamma f\left(x^{*}\right)-\right.$ $\left.A x^{*}, J\left(x_{n+1}-x^{*}\right)\right\rangle$. The above reduces to formula $\left\|x_{n+1}-x^{*}\right\|^{2} \leq\left(1-a_{n}\right)\left\|x_{n}-x^{*}\right\|^{2}+a_{n} b_{n}$. Observing control condition (C1) and noting (3.55), it is easily seen that $\sum_{n=1}^{\infty} a_{n}=\infty$ and $\limsup _{n \rightarrow \infty} b_{n} \leq 0$. By Lemma 2.6, we conclude that $\lim _{n \rightarrow \infty}\left\|x_{n}-x^{*}\right\|=0$. This completes the proof.

Corollary 3.3. Let $C$ be a nonempty bounded closed convex subset of a real Hilbert space $H$ with $C \pm C \subset C$ and $\tau=\{T(t): 0 \leq t<\infty\}$ be a nonexpansive semigroup on $C$ such that $F(\tau) \neq \emptyset$. Let $f: C \rightarrow C$ be a contraction mapping with a coefficient $\alpha \in(0,1)$ and $A$ be a strongly positive bounded linear operator with coefficient $\bar{\gamma}>0$ such that $0<\gamma<\bar{\gamma} / \alpha$. Let $\left\{\alpha_{n}\right\}_{n=1}^{\infty},\left\{\beta_{n}\right\}_{n=1}^{\infty},\left\{\delta_{n}\right\}_{n=1}^{\infty}$ 
be the sequences in $(0,1)$ and $\left\{t_{n}\right\}_{n=1}^{\infty}$ be a positive real divergent sequence such that $t_{n} \leq t_{n+1}$ for all $n \in \mathbb{N}$. Assume that the following control conditions that hold:

(C1) $\lim _{n \rightarrow \infty} \alpha_{n}=0$ and $\sum_{n=1}^{\infty} \alpha_{n}=\infty$;

(C2) $\sum_{n=1}^{\infty}\left|\alpha_{n+1}-\alpha_{n}\right|<\infty, \sum_{n=1}^{\infty}\left|\beta_{n+1}-\beta_{n}\right|<\infty$, and $\sum_{n=1}^{\infty}\left|\delta_{n+1}-\delta_{n}\right|<\infty$;

(C3) $\sum_{n=1}^{\infty} \sup _{z \in C}\left\{\left\|T(h) T\left(t_{n}\right) z-T\left(t_{n}\right) z\right\|\right\}<\infty$ for all $h \geq 0$.

Then the sequence $\left\{x_{n}\right\}$ defined by (1.13) converges strongly to the common fixed point $x^{*}$, where $x^{*}$ is the unique solution in $F(\tau)$ of the variational inequality (1.6).

Proof. From Theorem 3.2, if $X:=H$ is a real Hilbert space, we get the desired conclusion easily.

Corollary 3.4. Let $C$ be a nonempty bounded closed convex subset of a uniformly smooth Banach space $X$ which admit a weakly sequentially continuous duality mapping from $X$ into $X^{*}$ and $\tau=$ $\{T(t): 0 \leq t<\infty\}$ be a nonexpansive semigroup mapping on $C$ such that $F(\tau) \neq \emptyset$. Let $f: C \rightarrow C$ be a contraction mapping with a coefficient $\alpha \in(0,1)$. Let $\left\{\alpha_{n}\right\}_{n=1}^{\infty},\left\{\beta_{n}\right\}_{n=1}^{\infty},\left\{\delta_{n}\right\}_{n=1}^{\infty}$ be the sequences in $(0,1)$ and $\left\{t_{n}\right\}_{n=1}^{\infty}$ be a positive real divergent sequence such that $t_{n} \leq t_{n+1}$ for all $n \in \mathbb{N}$. Assume that the following control conditions hold:

(C1) $\lim _{n \rightarrow \infty} \alpha_{n}=0$ and $\sum_{n=1}^{\infty} \alpha_{n}=\infty$;

(C2) $\sum_{n=1}^{\infty}\left|\alpha_{n+1}-\alpha_{n}\right|<\infty, \sum_{n=1}^{\infty}\left|\beta_{n+1}-\beta_{n}\right|<\infty$, and $\sum_{n=1}^{\infty}\left|\delta_{n+1}-\delta_{n}\right|<\infty$;

(C3) $\sum_{n=1}^{\infty} \sup _{z \in C}\left\{\left\|T(h) T\left(t_{n}\right) z-T\left(t_{n}\right) z\right\|\right\}<\infty$ for all $h \geq 0$.

Then the sequence $\left\{x_{n}\right\}$ defined by

$$
\begin{gathered}
x_{1}=x \in C \text { chosen arbitrarily, } \\
y_{n}=\beta_{n} x_{n}+\left(1-\beta_{n}\right) T\left(t_{n}\right) x_{n} ; \\
x_{n+1}=\alpha_{n} f\left(x_{n}\right)+\delta_{n} x_{n}+\left(1-\delta_{n}-\alpha_{n}\right) y_{n}, \quad \forall n \geq 1,
\end{gathered}
$$

converges strongly to the fixed point $x^{*}$, where $x^{*}$ is the unique solution in $F(\tau)$ of the variational inequality (1.11).

Proof. Taking $A=I$ and $\gamma=1$, then (1.13) is reduced to (3.58).

Corollary 3.5 (Marino and $\mathrm{Xu}[12])$. Let $H$ be a real Hilbert space. Let $T$ be a nonexpansive mapping on $H$ such that $F(T) \neq \emptyset, f$ be a contraction mapping with a coefficient $\alpha \in(0,1)$ and $A$ be a strongly positive bounded linear operator with a coefficient $\bar{\gamma}>0$ such that $0<\gamma<\bar{\gamma} / \alpha$. Assume that the following control conditions hold:

(C1) $\lim _{n \rightarrow \infty} \alpha_{n}=0$ and $\sum_{n=1}^{\infty} \alpha_{n}=\infty$;

(C2) $\sum_{n=1}^{\infty}\left|\alpha_{n+1}-\alpha_{n}\right|<\infty$.

Then the sequence $\left\{x_{n}\right\}$ defined by

$$
\begin{gathered}
x_{0}=x \in H \text { chosen arbitrarily; } \\
x_{n+1}=\alpha_{n} \gamma f\left(x_{n}\right)+\left(I-\alpha_{n} A\right) T x_{n}, \quad \forall n \geq 0,
\end{gathered}
$$


converges strongly to the fixed point $x^{*}$, where $x^{*}$ is the unique solution in $F(T)$ of the variational inequality (1.6).

Proof. If $X:=H$ is a real Hilbert space, we get the desired conclusion easily. Taking $\delta_{n}=0$, $\beta_{n}=0$, and $T\left(t_{n}\right)=T$, then (1.13) is reduced to (3.59).

Now, we prove the following theorem, under certain different control conditions on the sequence $\left\{\alpha_{n}\right\},\left\{\beta_{n}\right\}$ and $\left\{\delta_{n}\right\}$.

Theorem 3.6. Let $C$ be a nonempty bounded closed convex subset of a uniformly smooth Banach space $X$ which admit a weakly sequentially continuous duality mapping from $X$ into $X^{*}$ with $C \pm C \subset C$ and $\tau=\{T(t): 0 \leq t<\infty\}$ be a nonexpansive semigroup on $C$ such that $F(\tau) \neq \emptyset$. Let $f: C \rightarrow C$ be a contraction mapping with a coefficient $\alpha \in(0,1)$ and $A$ be a strongly positive bounded linear operator with coefficient $\bar{\gamma}>0$ such that $0<\gamma<\bar{\gamma} / \alpha$. Let $\left\{\alpha_{n}\right\}_{n=1}^{\infty},\left\{\beta_{n}\right\}_{n=1}^{\infty},\left\{\delta_{n}\right\}_{n=1}^{\infty}$ be the sequences in $(0,1)$ and $\left\{t_{n}\right\}_{n=1}^{\infty}$ be a positive real divergent sequence such that $t_{n+1}=h+t_{n}$ for all $n \in \mathbb{N}$ and for all $h \geq 0$. Assume that the following control conditions are hold:

(C1) $\lim _{n \rightarrow \infty} \alpha_{n}=0$ and $\sum_{n=1}^{\infty} \alpha_{n}=\infty$;

(C2) $\lim _{n \rightarrow \infty}\left|\beta_{n+1}-\beta_{n}\right|=0$;

(C3) $0<\lim \inf _{n \rightarrow \infty} \delta_{n} \leq \lim \sup _{n \rightarrow \infty} \delta_{n}<1$.

Suppose that $\left\{T\left(t_{n}\right)\right\}$ is a u.a.r. nonexpansive semigroup on $C$. Then the sequence $\left\{x_{n}\right\}$ defined by (1.13) converges strongly to the common fixed point $x^{*}$, where $x^{*}$ is the unique solution in $F(\tau)$ of the variational inequality (3.2).

Proof. By using the same argument and techniques as those of Theorem 3.2, we note that $\left\{x_{n}\right\}$ is bounded, and so are the set $\left\{y_{n}\right\},\left\{f\left(x_{n}\right)\right\}$ and $\left\{T\left(t_{n}\right) x_{n}\right\}$. We will show that $\lim _{n \rightarrow \infty} \| x_{n+1}-$ $x_{n} \|=0$.

From Theorem 3.2, we note that

$$
\left\|y_{n+1}-y_{n}\right\| \leq\left\|x_{n+1}-x_{n}\right\|+\left\|T\left(t_{n+1}\right) x_{n}-T\left(t_{n}\right) x_{n}\right\|+\left|\beta_{n+1}-\beta_{n}\right|\left\|x_{n}-T\left(t_{n}\right) x_{n}\right\| .
$$

Define $x_{n+1}=\left(1-\delta_{n}\right) l_{n}+\delta_{n} x_{n}$. That is $l_{n}=\left(x_{n+1}-\delta_{n} x_{n}\right) /\left(1-\delta_{n}\right)$. Now, we compute $l_{n+1}-l_{n}$.

Then, we note that

$$
\begin{aligned}
l_{n+1}-l_{n} & =\frac{x_{n+2}-\delta_{n+1} x_{n+1}}{1-\delta_{n+1}}-\frac{x_{n+1}-\delta_{n} x_{n}}{1-\delta_{n}} \\
& =\frac{\alpha_{n+1} \gamma f\left(x_{n+1}\right)+\left(\left(1-\delta_{n+1}\right) I-\alpha_{n+1} A\right) y_{n+1}}{1-\delta_{n+1}}-\frac{\alpha_{n} \gamma f\left(x_{n}\right)+\left(\left(1-\delta_{n}\right) I-\alpha_{n} A\right) y_{n}}{1-\delta_{n}} \\
& =\frac{\alpha_{n+1}}{1-\delta_{n+1}}\left(\gamma f\left(x_{n+1}\right)-A y_{n+1}\right)+\frac{\alpha_{n}}{1-\delta_{n}}\left(A y_{n}-\gamma f\left(x_{n}\right)\right)+y_{n+1}-y_{n},
\end{aligned}
$$

one has

$$
\left\|l_{n+1}-l_{n}\right\| \leq \frac{\alpha_{n+1}}{1-\delta_{n+1}}\left\|\gamma f\left(x_{n+1}\right)-A y_{n+1}\right\|+\frac{\alpha_{n}}{1-\delta_{n}}\left\|A y_{n}-\gamma f\left(x_{n}\right)\right\|+\left\|y_{n+1}-y_{n}\right\| .
$$


Substituting (3.60) into (3.62), we have

$$
\begin{aligned}
\left\|l_{n+1}-l_{n}\right\| \leq & \frac{\alpha_{n+1}}{1-\delta_{n+1}}\left\|\gamma f\left(x_{n+1}\right)-A y_{n+1}\right\|+\frac{\alpha_{n}}{1-\delta_{n}}\left\|A y_{n}-\gamma f\left(x_{n}\right)\right\|+\left\|x_{n+1}-x_{n}\right\| \\
& +\left\|T\left(t_{n+1}\right) x_{n}-T\left(t_{n}\right) x_{n}\right\|+\left|\beta_{n+1}-\beta_{n}\right|\left\|x_{n}-T\left(t_{n}\right) x_{n}\right\| .
\end{aligned}
$$

By assuming that $t_{n+1}=h+t_{n}$, it follows that

$$
\begin{aligned}
\left\|l_{n+1}-l_{n}\right\|-\left\|x_{n+1}-x_{n}\right\| \leq & \frac{\alpha_{n+1}}{1-\delta_{n+1}}\left\|\gamma f\left(x_{n+1}\right)-A y_{n+1}\right\|+\frac{\alpha_{n}}{1-\delta_{n}}\left\|A y_{n}-\gamma f\left(x_{n}\right)\right\| \\
& +\left\|T(h) T\left(t_{n}\right) x_{n}-T\left(t_{n}\right) x_{n}\right\|+\left|\beta_{n+1}-\beta_{n}\right|\left\|x_{n}-T\left(t_{n}\right) x_{n}\right\| .
\end{aligned}
$$

Since $\left\{T\left(t_{n}\right)\right\}$ is a u.a.r. nonexpansive semigroup and $\lim _{n \rightarrow \infty} t_{n}=\infty$, then for all $h \geq 0$, we have

$$
\lim _{n \rightarrow \infty}\left\|T(h) T\left(t_{n}\right) x_{n}-T\left(t_{n}\right) x_{n}\right\| \leq \lim _{n \rightarrow \infty} \sup _{z \in C}\left\|T(h) T\left(t_{n}\right) z-T\left(t_{n}\right) z\right\|=0 .
$$

Moreover, since $\left\{x_{n}\right\},\left\{T\left(t_{n}\right) x_{n}\right\}$ are bounded, observing control conditions (C1), (C2), (C3), and noting (3.65) then, inequality (3.64) implies that

$$
\limsup _{n \rightarrow \infty}\left(\left\|l_{n+1}-l_{n}\right\|-\left\|x_{n+1}-x_{n}\right\|\right) \leq 0
$$

It follows by Lemma 2.5 that

$$
\lim _{n \rightarrow \infty}\left\|l_{n}-x_{n}\right\|=0
$$

Observing that

$$
\begin{aligned}
x_{n+1}-x_{n} & =\left(1-\delta_{n}\right) l_{n}+\delta_{n} x_{n}-x_{n} \\
& =\left(1-\delta_{n}\right)\left(l_{n}-x_{n}\right),
\end{aligned}
$$

and hence,

$$
\lim _{n \rightarrow \infty}\left\|x_{n+1}-x_{n}\right\|=0
$$

By the same proof in Theorem 3.2, we note that

$$
\left\|y_{n}-x_{n}\right\| \leq \frac{1}{1-\delta_{n}}\left(\left\|x_{n}-x_{n+1}\right\|+\alpha_{n}\left(\gamma\left\|f\left(x_{n}\right)\right\|+\left\|A y_{n}\right\|\right)\right) .
$$


Hence $\lim _{n \rightarrow \infty}\left\|y_{n}-x_{n}\right\|=0$. Moreover, by Theorem 3.2, we note that

$$
\lim _{n \rightarrow \infty}\left\|x_{n}-T\left(t_{n}\right) x_{n}\right\|=0
$$

Next, we show that $\lim _{n \rightarrow \infty}\left\|T(h) x_{n}-x_{n}\right\|=0$. We note that

$$
\begin{aligned}
\left\|T(h) x_{n}-x_{n}\right\| & \leq\left\|T(h) x_{n}-T(h) T\left(t_{n}\right) x_{n}\right\|+\left\|T(h) T\left(t_{n}\right) x_{n}-T\left(t_{n}\right) x_{n}\right\|+\left\|T\left(t_{n}\right) x_{n}-x_{n}\right\| \\
& \leq 2\left\|x_{n}-T\left(t_{n}\right) x_{n}\right\|+\left\|T(h) T\left(t_{n}\right) x_{n}-T\left(t_{n}\right) x_{n}\right\| .
\end{aligned}
$$

Since $\left\{T\left(t_{n}\right)\right\}$ is a u.a.r. nonexpansive semigroup, and noting (3.65), we have

$$
\lim _{n \rightarrow \infty}\left\|T(h) x_{n}-x_{n}\right\|=0
$$

for all $h \geq 0$. By using the same argument and techniques as those of Theorem 3.2, we have $\left\{x_{n}\right\}$ converges strongly to a common fixed point $x^{*} \in F(\tau)$. This completes the proof.

Corollary 3.7. Let $C$ be a nonempty bounded closed convex subset of a real Hilbert space $H$ with $C \pm C \subset C$ and let $\tau=\{T(t): 0 \leq t<\infty\}$ be a nonexpansive semigroup on $C$ such that $F(\tau) \neq \emptyset$. Let $f: C \rightarrow C$ be a contraction mapping with a coefficient $\alpha \in(0,1)$ and $A$ be a strongly positive bounded linear operator with coefficient $\bar{\gamma}>0$ such that $0<\gamma<\bar{\gamma} / \alpha$. Let $\left\{\alpha_{n}\right\}_{n=1}^{\infty},\left\{\beta_{n}\right\}_{n=1}^{\infty},\left\{\delta_{n}\right\}_{n=1}^{\infty}$ be the sequences in $(0,1)$ and $\left\{t_{n}\right\}_{n=1}^{\infty}$ be a positive real divergent sequence such that $t_{n+1}=h+t_{n}$ for all $n \in \mathbb{N}$ and for all $h \geq 0$. Assume that the following control conditions are hold:

$$
\begin{aligned}
& \text { (C1) } \lim _{n \rightarrow \infty} \alpha_{n}=0 \text { and } \sum_{n=1}^{\infty} \alpha_{n}=\infty ; \\
& \text { (C2) } \lim _{n \rightarrow \infty}\left|\beta_{n+1}-\beta_{n}\right|=0 \text {; } \\
& \text { (C3) } 0<\lim \inf _{n \rightarrow \infty} \delta_{n} \leq \limsup \sup _{n \rightarrow \infty} \delta_{n}<1 \text {. }
\end{aligned}
$$

Suppose that $\left\{T\left(t_{n}\right)\right\}$ is a u.a.r. nonexpansive semigroup on $C$. Then the sequence $\left\{x_{n}\right\}$ defined by (1.13) converges strongly to the fixed point $x^{*}$, where $x^{*}$ is the unique solution in $F(\tau)$ of the variational inequality (1.6).

Proof. From Theorem 3.6, if $X:=H$ is a real Hilbert space, we get the desired conclusion easily.

If we taking $A=I$ and $\gamma=1$, then (1.13) is reduced to (3.74). So, we obtain the following corollary.

Corollary 3.8. Let $C$ be a nonempty bounded closed convex subset of a uniformly smooth Banach space $X$ which admit a weakly sequentially continuous duality mapping from $X$ into $X^{*}$ and let $\tau=$ $\{T(t): 0 \leq t<\infty\}$ be a nonexpansive semigroup on $C$ such that $F(\tau) \neq \emptyset$. Let $f: C \rightarrow C$ be a contraction mapping with a coefficient $\alpha \in(0,1)$ and $\left\{\alpha_{n}\right\}_{n=1}^{\infty},\left\{\beta_{n}\right\}_{n=1}^{\infty},\left\{\delta_{n}\right\}_{n=1}^{\infty}$ be the sequences in 
$(0,1)$ and $\left\{t_{n}\right\}_{n=1}^{\infty}$ be a positive real divergent sequence such that $t_{n+1}=h+t_{n}$ for all $n \in \mathbb{N}$ and for all $h \geq 0$. Assume that the following control conditions hold:

(C1) $\lim _{n \rightarrow \infty} \alpha_{n}=0$ and $\sum_{n=1}^{\infty} \alpha_{n}=\infty$;

(C2) $\lim _{n \rightarrow \infty}\left|\beta_{n+1}-\beta_{n}\right|=0$;

(C3) $0<\lim \inf _{n \rightarrow \infty} \delta_{n} \leq \lim \sup _{n \rightarrow \infty} \delta_{n}<1$.

Suppose that $\left\{T\left(t_{n}\right)\right\}$ is a u.a.r. nonexpansive semigroup on $C$. Then the sequence $\left\{x_{n}\right\}$ defined by

$$
\begin{gathered}
x_{1}=x \in C \text { chosen arbitrarily; } \\
y_{n}=\beta_{n} x_{n}+\left(1-\beta_{n}\right) T\left(t_{n}\right) x_{n} ; \\
x_{n+1}=\alpha_{n} f\left(x_{n}\right)+\delta_{n} x_{n}+\left(1-\delta_{n}-\alpha_{n}\right) y_{n}, \quad \forall n \geq 1,
\end{gathered}
$$

converges strongly to the fixed point $x^{*}$, where $x^{*}$ is the unique solution in $F(\tau)$ of the variational inequality (1.11).

\section{Acknowledgment}

The authors would like to thank The National Research Council of Thailand (NRCT) and the Faculty of Science KMUTT for financial support.

\section{References}

[1] F. E. Browder," "Fixed-point theorems for noncompact mappings in Hilbert space," Proceedings of the National Academy of Sciences of the United States of America, vol. 53, no. 6, pp. 1272-1276, 1965.

[2] F. E. Browder, "Nonexpansive nonlinear operators in a Banach space," Proceedings of the National Academy of Sciences of the United States of America, vol. 54, no. 4, pp. 1041-1044, 1965.

[3] H. K. Xu, "Viscosity approximation methods for nonexpansive mappings," Journal of Mathematical Analysis and Applications, vol. 298, no. 1, pp. 279-291, 2004.

[4] K. Geobel and W. A. Kirk, Topics in Metric Fixed point Theory, vol. 28 of Cambridge Stud. Adv. Math., Cambridge University Press, Cambridge, UK, 1990.

[5] P. Kumam and K. Wattanawitoon, "A general composite explicit iterative scheme of fixed point solutions of variational inequalities for nonexpansive semigroups," Mathematical and Computer Modelling, vol. 53, pp. 998-1006, 2011.

[6] P. Sunthrayuth and P. Kumam, "A general iterative algorithm for the solution of variational inequalities for a nonexpansive semigroup in Banach spaces," Journal of Nonlinear Analysis and Optimization: Theory \& Applications, vol. 1, no. 1, pp. 139-150, 2010.

[7] H. K. Xu, "An iterative approach to quadratic optimization," Journal of Optimization Theory and Applications, vol. 116, no. 3, pp. 659-678, 2003.

[8] H. K. Xu, "Iterative algorithms for nonlinear operators," Journal of the London Mathematical Society, vol. 66, no. 1, pp. 240-256, 2002.

[9] I. Yamada, "The hybrid steepest descent method for the variational inequality problem of the intersection of fixed point sets for nonexpansive mappings," in Inherently Parallel Algorithms for Feasibility and Optimization, K. Butnariu, Y. Censor, and S. Reich, Eds., pp. 473-504, North-Holland, Amsterdam, The Netherlands, 2001.

[10] I. Yamada, N. Ogura, Y. Yamashita, and K. Sakaniwa, "Quadratic optimization of fixed points of nonexpansive mappings in Hilbert space," Numerical Functional Analysis and Optimization, vol. 19, no. 1-2, pp. 165-190, 1998.

[11] A. Moudafi, "Viscosity approximation methods for fixed-points problems," Journal of Mathematical Analysis and Applications, vol. 241, no. 1, pp. 46-55, 2000. 
[12] G. Marino and H. K. Xu, "A general iterative method for nonexpansive mappings in Hilbert spaces," Journal of Mathematical Analysis and Applications, vol. 318, no. 1, pp. 43-52, 2006.

[13] T. H. Kim and H. K. Xu, "Strong convergence of modified Mann iterations," Nonlinear Analysis: Theory, Methods and Applications, vol. 61, no. 1-2, pp. 51-60, 2005.

[14] Y. Yao, R. Chen, and J. C. Yao, "Strong convergence and certain control conditions for modified Mann iteration," Nonlinear Analysis: Theory, Methods and Applications, vol. 68, no. 6, pp. 1687-1693, 2008.

[15] D. Sahu and D. O'Regan, "Convergence theorems for semigroup-type families of non-self mappings," Rendiconti del Circolo Matematico di Palermo, vol. 57, no. 3, pp. 305-329, 2008.

[16] X. N. Li and J. S. Gu, "Strong convergence of modified Ishikawa iteration for a nonexpansive semigroup in Banach spaces," Nonlinear Analysis: Theory, Methods and Applications, vol. 73, pp. 10851092, 2010.

[17] G. Cai and C. S. Hu, "Strong convergence theorems of a general iterative process for a finite family of $\lambda_{i}$-strict pseudo-contractions in q-uniformly smooth Banach spaces," Computers and Mathematics with Applications, vol. 59, no. 1, pp. 149-160, 2010.

[18] W. Takahashi, Nonlinear Functional Analysis, Fixed Point Theory and Its Applications, Yokohama Publisher, Yokohama, Japan, 2000.

[19] R. E. Megginson, An Introduction to Banach Space Theory, Graduate Texts in Mathematics, Springer, New York, NY, USA, 1998.

[20] A. Aleyner and Y. Censor, "Best approximation to common fixed points of a semigroup of nonexpansiive operator," Journal of Nonlinear and Convex Analysis, vol. 6, no. 1, pp. 137-151, 2005.

[21] A. Aleyner and S. Reich, "An explicit construction of sunny nonexpansive retractions in Banach spaces," Fixed Point Theory and Applications, vol. 2005, no. 3, pp. 295-305, 2005.

[22] T. D. Benavides, G. L. Acedo, and H. K. Xu, "Construction of sunny nonexpansive retractions in banach spaces," Bulletin of the Australian Mathematical Society, vol. 66, no. 1, pp. 9-16, 2002.

[23] R. Chen and Y. Song, "Convergence to common fixed point of nonexpansive semigroups," Journal of Computational and Applied Mathematics, vol. 200, no. 2, pp. 566-575, 2007.

[24] Y. Song and S. Xu, "Strong convergence theorems for nonexpansive semigroup in Banach spaces," Journal of Mathematical Analysis and Applications, vol. 338, no. 1, pp. 152-161, 2008.

[25] T. Suzuki, "Strong convergence of Krasnoselskii and Mann's type sequences for one-parameter nonexpansive semigroups without Bochner integrals," Journal of Mathematical Analysis and Applications, vol. 305, no. 1, pp. 227-239, 2005.

[26] J. P. Gossez and E. Lami Dozo, "Some geometric properties related to the fixed points theory for nonexpansive mappings," Pacific Journal of Mathematics, vol. 40, pp. 565-573, 1972.

[27] J. S. Jung, "Iterative approaches to common fixed points of nonexpansive mappings in Banach spaces," Journal of Mathematical Analysis and Applications, vol. 302, no. 2, pp. 509-520, 2005.

[28] J. Schu, "Approximation of fixed points of asymptotically nonexpansive mappings," Proceedings of the American Mathematical Society, vol. 112, no. 1, pp. 143-151, 1991.

[29] L. S. Liu, "Ishikawa and Mann iterative processes with errors for nonlinear strongly accretive mappings in Banach spaces," Journal of Mathematical Analysis and Applications, vol. 194, no. 1, pp. 114-125, 1995.

[30] K. Aoyama, Y. Kimura, W. Takahashi, and M. Toyoda, "Approximation of common fixed points of a countable family of nonexpansive mappings in a Banach space," Nonlinear Analysis: Theory, Methods and Applications, vol. 67, no. 8, pp. 2350-2360, 2007. 


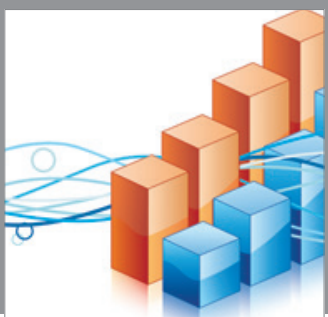

Advances in

Operations Research

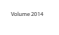

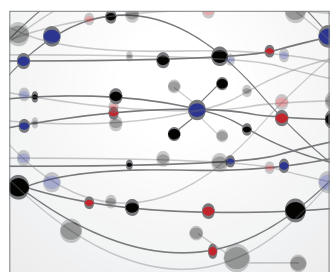

\section{The Scientific} World Journal
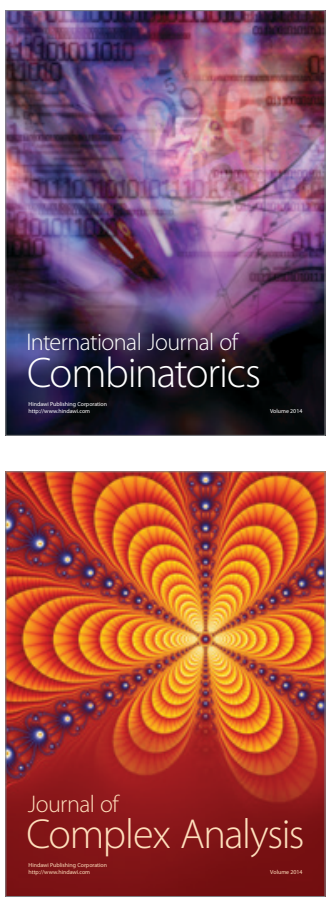

International Journal of

Mathematics and

Mathematical

Sciences
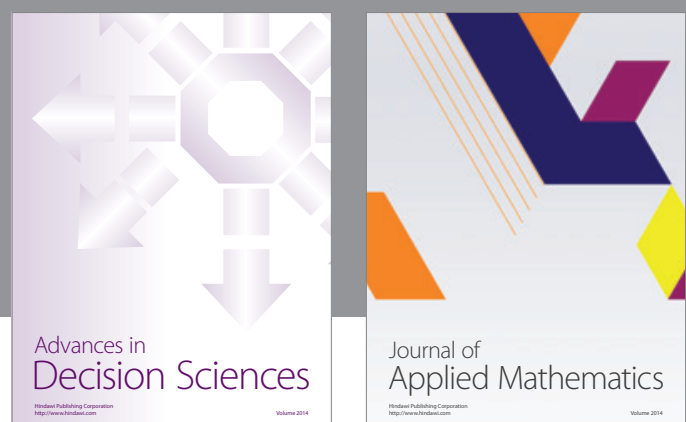

Journal of

Applied Mathematics
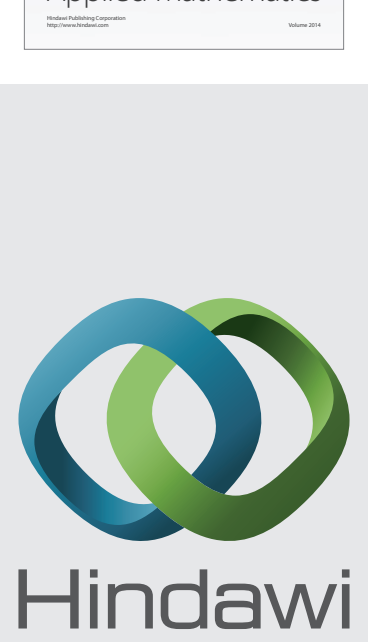

Submit your manuscripts at http://www.hindawi.com
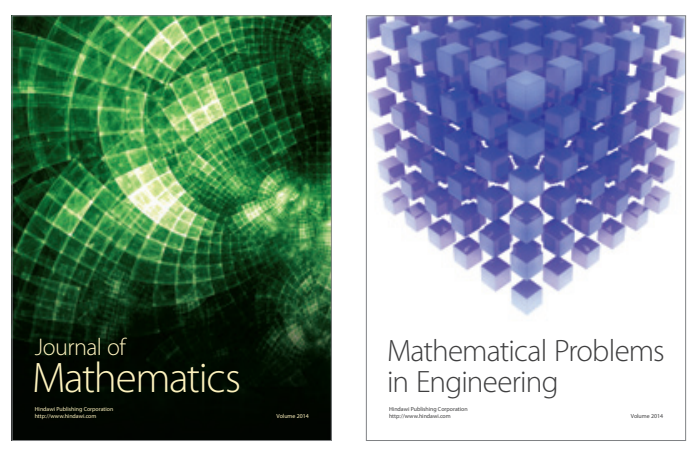

Mathematical Problems in Engineering
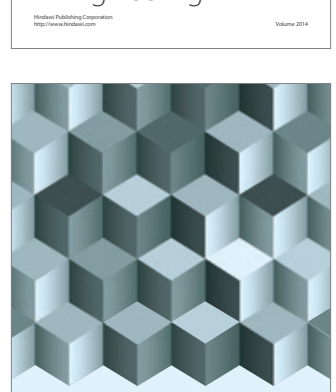

Journal of

Function Spaces
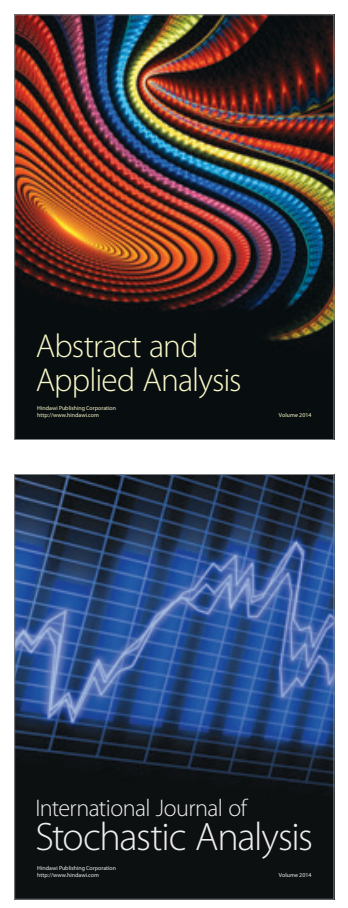

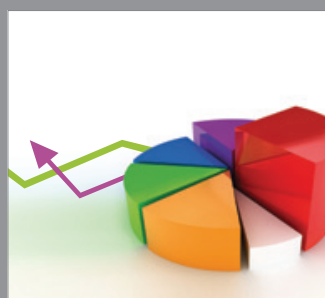

ournal of

Probability and Statistics

Promensencen
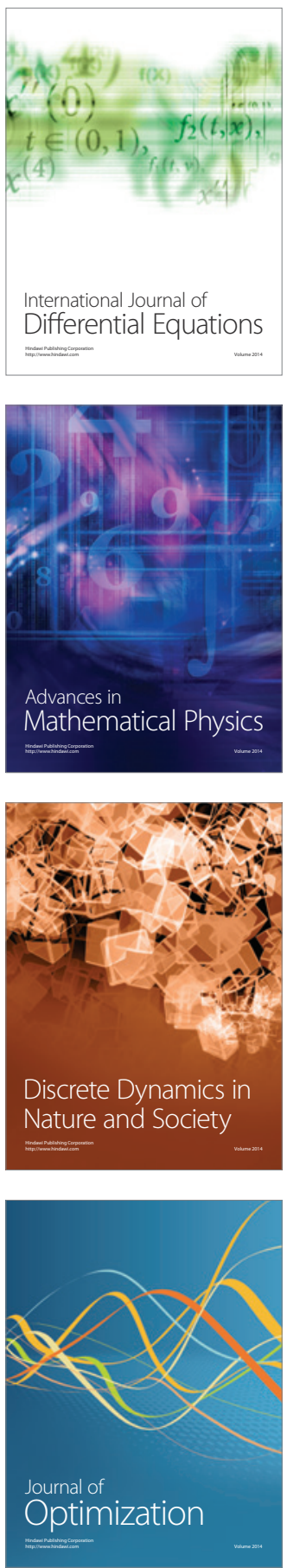